\title{
Non-Symmetric Matrix Riccati Equations
}

\author{
G. Freiling and G. Jank
}

\begin{abstract}
We prove a fundamental representation formula for all solutions of the matrix Riccati differential equation and of the corresponding algebraic Riccati equation. This formula contains the complete information on the phase portrait of the matrix equation and on the structure of the set $\Gamma$ of all solutions of the corresponding algebraic equation. In particular we describe all constant, periodic and almost-periodic solutions of the matrix Riccati differential equation. Further we give an application of the fundamental representation formula to the investigation of non-autonomous Riccati equations.
\end{abstract}

Keywords: Matrix Riccati differential equation, algebraic Riccati equation, asymptotic properties

AMS subject classification: 34 A 46, 34 C 25, 34 C 27, 34 E 10, 49 J 15, 93 C 15

\section{Introduction}

In this paper we study the matrix Riccati differential equation

$$
\dot{W}=B_{21}+B_{22} W-W B_{11}-W B_{12} W \quad(t \in C \quad \text { or } t \in \mathbb{R})
$$

(we will write $(\mathrm{RDE})_{\boldsymbol{C}}$ and $(\mathrm{RDE})_{\mathbb{R}}$ for $t \in \boldsymbol{C}$ and $t \in \mathbb{R}$, respectively) and the corresponding algebraic Riccati equation

$$
0=B_{21}+B_{22} W-W B_{11}-W B_{12} W
$$

where $W(t)$ (or $W$ ) is a complex $m \times n$ matrix and where $B_{11}, B_{12}, B_{21}, B_{22}$ are constant complex matrices of dimensions $n \times n, n \times m, m \times n$ and $m \times m$, respectively. The matrix Riccati equation - especially $(\mathrm{RDE})_{\mathbb{R}}$ and (ARE) for $m=n$ - plays an important role in many branches of applied mathematics, notably in variational theory and the allied areas of optimal control and filtering, invariant imbedding, spectral factorization and dynamic programming (see $[5,8,14,21]$ ). Non-square matrix Riccati equations appear for example in Nash and Stackelberg control problems, where the properties of Riccati equations determine the existence of the optimal open-loop strategies (see $[1,18,28]$ for further references). On the other hand (RDE) is also of mathematical interest, since it is

G. Freiling: Rhein.-Westfäl. Techn. Hochschule (RWTH), Lehrstuhl II für Math., Templergraben 55, D - 52056 Aachen

G. Jank: Rhein.-Westfäl. Techn. Hochschule (RWTH), Lehrstuhl II für Math., Templergraben 55, D - 52056 Aachen

ISSN 0232-2064 / $\$ 2.50$ (C) Heldermann Verlag 
the description in local coordinates of the differential equation on a Graßmann manifold, whose flow is given by the action of a one-parameter subgroup of $G l(n+m, \boldsymbol{C})$ (see $[10,22,27])$.

In Section 2 of this paper we derive a fundamental representation formula (2.10) for the solutions of equation (RDE). This formula contains the complete information on the phase portrait of (RDE) and in particular on the structure of the set $\Gamma$ of all solutions of equation (ARE) (see Sections 3 and 4). In the last part of this note we give an application of the fundamental representation formula to the investigation of non-autonomous Riccati equations.

\section{The fundamental representation formula}

In this section we describe the connection between equation (RDE) and the corresponding linear differential equation (L) (see [21: p. 11]), and we derive a detailed formula for the general solution of equation (RDE).

Let

$$
B=\left(\begin{array}{ll}
B_{11} & B_{12} \\
B_{21} & B_{22}
\end{array}\right) \quad \text { and } \quad Y=\left(\begin{array}{l}
Q \\
P
\end{array}\right)
$$

with complex $n \times n$ and $m \times n$ matrix functions $Q$ and $P$, respectively. Then equation $(\mathrm{RDE})$ and the linear system of differential equations

$$
\dot{Y}=B Y \quad(t \in \boldsymbol{C})
$$

are equivalent in the following sense:

(i) Let $I_{n} \in \boldsymbol{C}^{n \times n}$ be the unit matrix and let $W$ be a solution of equation (RDE). If $Q$ is the unique solution of the initial value problem

$$
\begin{aligned}
\dot{Q} & =\left(B_{11}+B_{12} W(t)\right) Q \\
Q\left(t_{0}\right) & =I_{n} \quad\left(t_{0} \in G \subset \boldsymbol{C}\right)
\end{aligned}
$$

and $P(t)=W(t) Q(t)$, then $Y=\left(\begin{array}{l}Q \\ P\end{array}\right)$ is a solution of equation (L). then

(ii) If $Y=\left(\begin{array}{c}Q \\ P\end{array}\right)$ is a solution of equation (L) such that $Q(t)$ is regular for $t \in G \subset \boldsymbol{C}$,

$$
W: G \rightarrow C^{m \times n}, \quad t \longrightarrow P(t) Q^{-1}(t)=W(t)
$$

is a solution of equation ( $\mathrm{RDE})$.

Let

$$
V=\left(v_{1}, \ldots, v_{n+m}\right) \in \boldsymbol{C}^{(n+m) \times(n+m)}
$$

be the matrix defined by a Jordan basis of generalized eigenvectors of $B$ such that

$$
V^{-1} B V=J=\operatorname{diag}\left(J_{1}, \ldots, J_{p}\right)=\left(\begin{array}{ccc}
\lambda_{1} & * & 0 \\
\ddots & \ddots & * \\
0 & \ddots & \lambda_{n+m}
\end{array}\right)
$$


with $* \in\{0,1\}$ and (without loss of generality)

$$
\operatorname{Re} \lambda_{1} \leq \operatorname{Re} \lambda_{2} \leq \ldots \leq \operatorname{Re} \lambda_{n+m}
$$

and where $J$ is a Jordan canonical form of $B$ with Jordan blocks

$$
J_{\nu}=\left(\begin{array}{cccc}
\mu_{\nu} & 1 & \ldots & 0 \\
0 & \mu_{\nu} & \ddots & \vdots \\
\vdots & \ddots & \ddots & 1 \\
0 & \ldots & 0 & \mu_{\nu}
\end{array}\right) \in \boldsymbol{C}^{j_{\nu} \times j_{\nu}} \quad\left(\begin{array}{c}
1 \leq \nu \leq p \\
\mu_{\nu} \in\left\{\lambda_{1}, \ldots, \lambda_{n+m}\right\}
\end{array}\right)
$$

If

$$
C=\left(\begin{array}{c}
c_{1} \\
\vdots \\
c_{n+m}
\end{array}\right)=\left(\begin{array}{ccc}
c_{11} & \cdots & c_{1 n} \\
\vdots & & \vdots \\
c_{n+m, 1} & \ldots & c_{n+m, n}
\end{array}\right) \in \boldsymbol{C}^{(n+m) \times n}
$$

and

$$
\left(x_{1}(t), \ldots, x_{n+m}(t)\right) \operatorname{diag}\left(e^{\lambda_{1} t}, \ldots, e^{\lambda_{n+m} t}\right):=V e^{J t},
$$

then an arbitrary $((n+m) \times n)$-matrix solution $Y=Y(t)=Y(t ; C)$ of equation (L) has the form

$$
Y(t)=V e^{J t} C=\left(\begin{array}{c}
Q(t) \\
P(t)
\end{array}\right)=\left(\begin{array}{c}
Q(t ; C) \\
P(t ; C)
\end{array}\right)
$$

with

$$
Q(t) \in \boldsymbol{C}^{n \times n}, \quad P(t) \in \boldsymbol{C}^{m \times n}, \quad Y(0)=V C=\left(\begin{array}{l}
Q_{0}(C) \\
P_{0}(C)
\end{array}\right) .
$$

Further, to every Jordan block $J_{\nu}(1 \leq \nu \leq p)$ there correspond $j_{\nu}$ solution vectors of the form

$$
\begin{aligned}
\hat{y}_{j_{1}+\ldots+j_{\nu-1}+1}(t) & =e^{\mu_{\nu} t} v_{j_{1}+\ldots+j_{\nu-1}+1}=: e^{\mu_{\nu} t} x_{j_{1}+\ldots+j_{\nu-1}+1}(t) \\
& \vdots \\
\hat{y}_{j_{1}+\ldots+j_{\nu}}(t) & =e^{\mu_{\nu} t} \sum_{i=1}^{j_{\nu}} \frac{t^{j_{\nu}-i}}{\left(j_{\nu}-i\right) !} v_{j_{1}+\ldots+j_{\nu-1}+i}=: e^{\mu_{\nu} t} x_{j_{1}+\ldots+j_{\nu}}(t)
\end{aligned}
$$

$\left(1 \leq \nu \leq p\right.$ and $\left.j_{0}:=0\right)$. Let

$$
x_{\nu}(t)=\left(\begin{array}{c}
x_{\nu, 1}(t) \\
\vdots \\
x_{\nu, n+m}(t)
\end{array}\right) \quad(1 \leq \nu \leq n+m)
$$

be the polynomials defined by $(2.5), 1 \leq j \leq n$ and $1 \leq \ell \leq m$. Then we set

$$
\tilde{x}_{\nu}=\left(\begin{array}{c}
x_{\nu 1} \\
\vdots \\
x_{\nu n}
\end{array}\right), \quad x_{\nu}(\ell, j)=\left(x_{\nu 1}, \ldots, x_{\nu, j-1}, x_{\nu, n+\ell}, x_{\nu, j+1}, \ldots, x_{\nu n}\right)^{\top} .
$$


Similarly we define $\tilde{v}_{\nu}$ and $v_{\nu}(\ell, j)$. Using these notations we infer from (2.4)

$$
\begin{aligned}
Q(t) & =\left(\sum_{\nu=1}^{n+m} c_{\nu 1} e^{\lambda_{\nu} t} \tilde{x}_{\nu}(t), \ldots, \sum_{\nu=1}^{n+m} c_{\nu n} e^{\lambda_{\nu} t} \tilde{x}_{\nu}(t)\right) \\
& =\left(\begin{array}{c}
\sum_{k=1}^{n+m} x_{k 1}(t) e^{\lambda_{k} t} c_{k} \\
\vdots \\
\sum_{k=1}^{n+m} x_{k n}(t) e^{\lambda_{k} t} c_{k}
\end{array}\right)=\left(\begin{array}{ccc}
Q_{11}(t) & \ldots & Q_{1 n}(t) \\
\vdots & & \vdots \\
Q_{n 1}(t) & \ldots & Q_{n n}(t)
\end{array}\right)
\end{aligned}
$$

and

$$
\begin{aligned}
P(t) & =\left(\sum_{\nu=1}^{n+m} c_{\nu 1} e^{\lambda_{\nu} t}\left(\begin{array}{c}
x_{\nu, n+1}(t) \\
\vdots \\
x_{\nu, n+m}(t)
\end{array}\right), \ldots, \sum_{\nu=1}^{n+m} c_{\nu n} e^{\lambda_{\nu} t}\left(\begin{array}{c}
x_{\nu, n+1}(t) \\
\vdots \\
x_{\nu, n+m}(t)
\end{array}\right)\right) \\
& =\left(\begin{array}{c}
\sum_{k=1}^{n+m} x_{k, n+1}(t) e^{\lambda_{k} t} c_{k} \\
\vdots \\
\sum_{k=1}^{n+m} x_{k, n+m}(t) e^{\lambda_{k} t} c_{k}
\end{array}\right) .
\end{aligned}
$$

Consequently we obtain, using the multilinearity of the determinant,

$$
\begin{aligned}
\operatorname{det} Q(t)= & \sum_{k_{1}=1}^{n+m} \ldots \sum_{k_{n}=1}^{n+m} e^{\left(\lambda_{k_{1}}+\ldots+\lambda_{k_{n}}\right) t} c_{k_{1}} 1 \ldots c_{k_{n} n}\left|\tilde{x}_{k_{1}}(t), \ldots, \tilde{x}_{k_{n}}(t)\right| \\
= & \sum_{1 \leq k_{1}<k_{2}<\ldots<k_{n} \leq n+m} e^{\left(\lambda_{k_{1}}+\ldots+\lambda_{k_{n}}\right) t}\left|\tilde{x}_{k_{1}}(t), \ldots, \tilde{x}_{k_{n}}(t)\right| \\
& \times\left(\sum_{\pi \in \sigma_{n}} \operatorname{sign} \pi \cdot c_{k_{\pi}(1)} 1 \ldots c_{k_{\pi(n)} n}\right) \\
= & \sum_{1 \leq k_{1}<k_{2}<\ldots<k_{n} \leq n+m} e^{\left(\lambda_{k_{1}}+\ldots+\lambda_{k_{n}}\right) t}\left|\tilde{x}_{k_{1}}(t), \ldots, \tilde{x}_{k_{n}}(t)\right|\left|\begin{array}{c}
c_{k_{1}} \\
\vdots \\
c_{k_{n}}
\end{array}\right| .
\end{aligned}
$$

We recall that

$$
\left|\tilde{x}_{k_{1}}(t), \therefore, \tilde{x}_{k_{n}}(t)\right|=\left|\tilde{v}_{k_{1}}, \ldots, \tilde{v}_{k_{n}}\right|
$$

is constant if $B$ is semisimple, otherwise these determinants are polynomials with coefficients of the form $\left|\tilde{v}_{\nu_{1}}, \ldots ; \tilde{v}_{\nu_{n}}\right|$.

Since $\operatorname{det} Q(t)$ is an exponential sum with polynomials as coefficients, the asymptotical distribution of the zeros of $\operatorname{det} Q(t)$ is well known (see Lemma 1, [4] and [16]).

For the evaluation of $\left(P Q^{-1}\right) \ell \alpha$, the element in the $\ell$-th row and $\alpha$-th column of $P Q^{-1}$, we use (2.6) - (2.8). From

$$
Q_{\alpha \beta}(t)=\sum_{k=1}^{n+m} c_{k \beta} e^{\lambda_{k} t} x_{k \alpha}(t)
$$


and

$$
Q^{-1}(t)=\frac{1}{\operatorname{det} Q(t)}\left(\begin{array}{ccc}
a_{11}(t) & \ldots & a_{1 n}(t) \\
\vdots & & \vdots \\
a_{n 1}(t) & \ldots & a_{n n}(t)
\end{array}\right)
$$

where $a_{i j}(t)$ is the minor of $Q_{j i}(t)$ with respect to $Q(t)$, we get for $\operatorname{det} Q(t) \neq 0$ (as with $(2.8))$

$$
\begin{aligned}
& \operatorname{det} Q(t)\left(P Q^{-1}(t)\right) \ell \alpha \\
& =\sum_{k=1}^{n+m} e^{\lambda_{k} t} x_{k, n+\ell}\left(c_{k 1}, \ldots, c_{k n}\right)\left(\begin{array}{c}
a_{1 \alpha} \\
\vdots \\
a_{n \alpha}
\end{array}\right) \\
& =\sum_{k=1}^{n+m} e^{\lambda_{k} t} x_{k, n+\ell}\left|\begin{array}{ccc}
Q_{11}(t) & \ldots & Q_{1 n}(t) \\
\vdots & & \vdots \\
Q_{\alpha-1,1}(t) & \ldots & Q_{\alpha-1, n}(t) \\
c_{k 1} & \ldots & c_{k n} \\
Q_{\alpha+1,1}(t) & \ldots & Q_{\alpha+1, n}(t) \\
\vdots & & \vdots \\
Q_{n 1}(t) & \ldots & Q_{n n}(t)
\end{array}\right| \\
& =\sum_{k=1}^{n+m} e^{\lambda_{k} t} x_{k, n+\ell} \sum_{\nu_{1}=1}^{n+m} \ldots \sum_{\nu_{\alpha}=1}^{n+m} \sum_{\nu_{\alpha+1}=1}^{n+m} \ldots \sum_{\nu_{n}=1}^{n+m} e^{\lambda_{\nu_{1}}+\ldots+\lambda_{\nu_{\alpha}-1}+\lambda_{\nu_{\alpha}+1}+\ldots+\lambda_{\nu n}} \\
& \times x_{\nu_{1}, 1}(t) \ldots x_{\nu_{\alpha-1}, \alpha-1}(t) x_{\nu_{\alpha+1}, \alpha+1}(t) \ldots x_{\nu_{n}, n}(t)\left|\begin{array}{ccc}
c_{\nu_{1} 1} & \ldots & c_{\nu_{1} n} \\
\vdots & & \vdots \\
c_{\nu_{\alpha}-1,1} & \ldots & c_{\nu_{\alpha-1}, n} \\
c_{k 1} & \ldots & c_{k n} \\
c_{\nu_{\alpha+1}, 1} & \ldots & c_{\nu_{\alpha+1}, n} \\
\vdots & & \vdots \\
c_{\nu_{n} 1} & \ldots & c_{\nu_{n} n}
\end{array}\right| \\
& =\sum_{1 \leq \nu_{1}<\nu_{2}<\ldots<\nu_{n} \leq n+m} e^{\left(\lambda_{\nu_{1}}+\ldots+\lambda_{\nu_{n}}\right) t}\left|x_{\nu_{1}}(\ell, \alpha), \ldots, x_{\nu_{n}}(\ell, \alpha)\right|(t)\left|\begin{array}{c}
c_{\nu_{1}} \\
\vdots \\
c_{\nu_{n}}
\end{array}\right| \text {. }
\end{aligned}
$$

We summarize the preceding analysis as a theorem.

Theorem 1 (Fundamental representation formula for the solutions of (RDE)): Let $Y(\cdot ; C), Q_{0}(C)$ and $P_{0}(C)$ be defined by (2.4). Further let $Q_{0}(C)$ be regular and $W_{C}=$ $P_{0}(C) Q_{0}(C)^{-1}$. Then

$$
\begin{aligned}
W\left(; W_{C}\right): \boldsymbol{C} \backslash\{t \in \boldsymbol{C} \mid \operatorname{det} Q(t)=0\} & \longrightarrow \boldsymbol{C}^{m \times n} \\
t & \longmapsto P(t) Q(t)^{-1}=W\left(t ; W_{C}\right)
\end{aligned}
$$


is a solution of equation ( $\mathrm{RDE})$ with $W\left(0 ; W_{C}\right)=W_{C}$ and

$$
\begin{aligned}
& w_{\ell a}\left(t ; W_{C}\right)= \\
& \frac{\sum_{1 \leq k_{1}<\ldots<k_{n} \leq n+m} e^{\left(\lambda_{k_{1}}+\ldots+\lambda_{k_{n}}\right) t}\left|x_{k_{1}}(\ell, \alpha), \ldots, x_{k_{n}}(\ell, \alpha)\right|(t)\left|\begin{array}{c}
c_{k_{1}} \\
\vdots \\
c_{k_{n}}
\end{array}\right|}{\sum_{1 \leq k_{1}<\ldots<k_{n} \leq n+m} e^{\left(\lambda_{k_{1}}+\ldots+\lambda_{k_{n}}\right) t}\left|\tilde{x}_{k_{1}}, \ldots, \tilde{x}_{k_{n}}\right|(t)\left|\begin{array}{c}
c_{k_{1}} \\
\vdots \\
c_{k_{n}}
\end{array}\right|}
\end{aligned}
$$

$(1 \leq \ell \leq m, 1 \leq \alpha \leq n)$.

Remark 1. (i) Obviously any solution $W\left(\cdot ; W_{C}\right)$. of equation (RDE) defines a meromorphic matrix function with poles at most in the zeros of $\operatorname{det} Q(t)$. This solution $W\left(\cdot ; W_{C}\right)$ is called the solution corresponding to $S_{0}=\operatorname{span}(V C)$, the column space of $V C$. The coefficients $\left|c_{k_{1}} \cdots c_{k_{n}}\right|^{\top}$ appearing in formula (2.10) are the so-called Plücker coordinates of $C$ (or of the n-dimensional subspace span $C$ ). These coefficients and also the coefficients

$$
\left|\tilde{x}_{k_{1}}, \ldots, \tilde{x}_{k_{n}}\right|(t) \quad \text { and } \quad\left|x_{k_{1}}(\ell, \alpha), \ldots, x_{k_{n}}(\ell, \alpha)\right|(t)
$$

are coupled (see Lemmas 1 and 3), which has strong influence on the behaviour of the solutions of equation (RDE) and on the structure of the set of all solutions of equation (ARE).

(ii) If the coefficients $B_{i j}$ in equation (RDE) are functions of $t$ and/or of some parameter $\rho$ and if the differential equation (L) has a $(n+m) \times(n+m)$ fundamental matrix solution of the form

$$
\left(z_{1}(t, \rho), \ldots, z_{n+m}(t, \rho)\right) \operatorname{diag}\left(e^{\lambda_{1}(t, \rho)}, \ldots, e^{\lambda_{n+m}(t, \rho)}\right)
$$

then the statement of Theorem 1 remains true for the non-autonomous or parameter dependent matrix Riccati equation, if we replace the vector polynomials $x_{k}(t)$ in formula (2.10) by the functions $z_{k}(t, \rho)$ and $\lambda_{k} t$ by $\lambda_{k}(t, \rho)(1 \leq k \leq n+m)$.

In the special case of T-periodic coefficients $B_{i j}(t)$ equation (L) has, according to the Floquet-Lyapunov theorem, a fundamental system of solutions $\hat{y}_{1}, \ldots, \hat{y}_{n+m}$ of the form (2.5) with $\mathrm{T}$-periodic vector functions $v_{1}, \ldots, v_{n+m}$ (instead of eigenvectors). If we define the polynomials $x_{1}, \ldots, x_{n+m}$ (with T-periodic coefficients) formally as with (2.5), then the fundamental representation formula (2.10) can also be used in the $\mathrm{T}$ periodic case.

(iii) As far as we know the fundamental representation formula (2.10) for the general solution of equation (RDE) is in this detailed form new even in the autonomous case. Obviously formula (2.10) contains the complete information on the phase portrait of equation (RDE) and particularly the complete information on all the solutions of equation (ARE). 
The properties of the solutions of equations (RDE) and (ARE) are very important for various branches of mathematics, therefore these equations have received considerable attention.

Readers who are interested in a detailed description of the geometry of the solutions of the most important special cases of equations (ARE) and (RDE) and of the so-called extended Riccati equation (ERDE) (see [27]) are referred to the papers of Callier and Willems [7], Shayman [23 - 27] and Hermann and Martin [10, 11] on this topic and to the literature cited therein.

In the next section we use formula $(2.10)$ to study the geometry of the solutions of equations (ARE) and (RDE). We focus our investigations mainly to those subcases which have not yet been considered in detail in $[18,23-27,28]$ - in this sense our results complement those of Shayman and also those of Medanic [18] and Telford and Moore [28].

\section{The solutions of the algebraic Riccati equation}

In this section we use the representation formula (2.10) to derive a parametrization and a geometric description of the set $\Gamma$ of all solutions of equation (ARE).

3.1 Notations. For the formulation of our results we introduce the following notations.

a) For

$$
\Lambda=\left(\lambda_{1}, \ldots, \lambda_{n+m}\right) \quad \text { and } \quad a=\left(a_{1}, \ldots, a_{n+m}\right) \in\{0,1\}^{n+m}
$$

we set

$$
\zeta(a)=\langle\Lambda, a\rangle=\sum_{\nu=1}^{n+m} a_{\nu} \lambda_{\nu}, \quad R(a)=\operatorname{Re} \zeta(a), \quad I(a)=\operatorname{Im} \zeta(a) .
$$

b) Let $N=\left(\begin{array}{c}n+m \\ n\end{array}\right)$ and let

$$
A=\left\{a^{1}, \ldots, a^{N}\right\} \subset\{0,1\}^{n+m}
$$

be the set with

$$
a^{j}=\left(a_{1}^{j}, \ldots, a_{n+m}^{j}\right) \quad \text { and } \quad a_{i}^{j}= \begin{cases}1 & \text { for } i \in\left\{\nu_{1}^{j}, \ldots, \nu_{n}^{j}\right\} ; \nu_{1}^{j}<\ldots<\nu_{n}^{j} \\ 0 & \text { else }\end{cases}
$$

In the sequel we assume that the $N$ elements of $A$ are enumerated such that

$$
j<k \Longleftrightarrow\left\{\begin{array}{l}
\text { either } \operatorname{Re}\left(\zeta\left(a^{j}\right) e^{i \phi}\right)<\operatorname{Re}\left(\zeta\left(a^{k}\right) e^{i \phi}\right) \text { for } 0<\phi \leq \varepsilon_{0} \\
\text { or } \zeta\left(a^{j}\right)=\zeta\left(a^{k}\right) \text { and }\left(\nu_{1}^{j}, \ldots, \nu_{n}^{j}\right) \leq \operatorname{lex}\left(\nu_{1}^{k}, \ldots, \nu_{n}^{k}\right)
\end{array}\right.
$$

for some $\varepsilon_{0}>0$, where $\leq_{\text {lex }}$ means lexocographical ordering. 
c) For

$$
a^{j} \in A \quad \text { and } \quad C=\left(\begin{array}{c}
c_{1} \\
\vdots \\
c_{n+m}
\end{array}\right) \in \boldsymbol{C}^{(n+m) \times n}
$$

and with the polynomials $x_{\nu}$ defined in (2.5) we set

$$
\begin{aligned}
p\left(t, a^{j}\right) & =\left|\tilde{x}_{\nu_{1}^{j}}(t), \ldots, \tilde{x}_{\nu_{n}^{j}}(t)\right| \\
p_{\ell \alpha}\left(t, a^{j}\right) & =\left|x_{\nu_{1}^{j}}(\ell, \alpha), \ldots, x_{\nu_{n}^{j}}(\ell, \alpha)\right|(t) \quad(1 \leq \ell \leq m ; 1 \leq \alpha \leq n) \\
D\left(a^{j}, C\right) & =\left|c_{\nu_{1}^{j}} \cdots c_{\nu_{n}^{j}}\right|^{\top} .
\end{aligned}
$$

With these abbreviations formula (2.10) can be written in the form

$$
w_{\ell, \alpha}\left(t ; W_{C}\right)=\frac{\sum_{a^{j} \in A} e^{t \zeta\left(a^{j}\right)} p_{\ell \alpha}\left(t, a^{j}\right) D\left(a^{j}, C\right)}{\sum_{a^{j} \in A} e^{t \zeta\left(a^{j}\right)} p\left(t, a^{j}\right) D\left(a^{j}, C\right)} .
$$

The following lemma and Lemma 3 indicate the type of coupling of the coefficients appearing in formulas (2.10) and (3.2).

Lemma 1. The following statements are true.

a) Let $1 \leq k_{1}<k_{2}<\cdots<k_{n} \leq n+m, j \in\{1, \ldots, n+m\} \backslash\left\{k_{1}, \ldots, k_{n}\right\}$ and $\left|\tilde{v}_{k_{1}}, \cdots, \tilde{v}_{k_{n}}\right| \neq 0$. Then at least one of the determinants

$$
\left|\tilde{v}_{j}, \tilde{v}_{k_{2}}, \ldots, \tilde{v}_{k_{n}}\right| \quad \text { or }\left|v_{j}(\ell, \alpha), v_{k_{2}}(\ell, \alpha), \ldots, v_{k_{n}}(\ell, \alpha)\right| \quad(1 \leq \ell \leq m ; 1 \leq \alpha \leq n)
$$

is non-zero.

b) Let $a^{j} \in A$ and let $p\left(t, a^{j}\right)=c t^{k} \quad(c \neq 0)$ be a monomial of degree $k \geq 1$. Then at least one of the polynomials

$$
p_{\ell \alpha}\left(t, a^{j}\right) \quad(1 \leq \ell \leq m ; 1 \leq \alpha \leq n)
$$

is not identically zero and not a monomial of degree $k$.

Proof. The matrix $D_{0}=\left(v_{j}, v_{k_{2}}, \ldots, v_{k_{n}}\right)$ has rank $n$ and by assumption $D_{1}=$ $\left(\tilde{v}_{k_{2}}, \ldots, \tilde{v}_{k_{n}}\right)$ has rank $n-1$. Let the first $n-1$ rows of $D_{1}$ be linearly independent - otherwise we proceed similarly. Then we can transform $D_{0}$ by elementary column operations into the form

$$
\left(\begin{array}{ll}
0 & F \\
b & D
\end{array}\right) \quad \text { where } b \in C^{m+1} \backslash\{0\} \text { and } F \in C^{(n-1) \times(n-1)} \text { is regular. }
$$

This proves assertion a).

Assertion b) (which can also be obtained from the results of [19]) follows with assertion a) from the definition of the polynomials $p\left(t, a^{j}\right)$ and $p_{\ell \alpha}\left(t, a^{j}\right)$. Notice the special form of the vector polynomials $x_{k}(t)$ in (2.5) 
3.2 The asymptotic behaviour of $W\left(r e^{i \varphi} ; W_{C}\right)$ for $r \rightarrow \infty$. The complex plane is divided into $2 h$ sectors

$$
S_{k}=\left\{t \in C \mid \varphi_{k-1} \leq \arg t \leq \varphi_{k}\right\} \quad(1 \leq k \leq 2 h)
$$

where the following holds true:

(i) $\varphi_{0} \leq 0<\varphi_{1}<\varphi_{2}<\ldots<\varphi_{2 h}=\varphi_{0}+2 \pi \leq 2 \pi$.

(ii) For $1 \leq k \leq 2 h$ there is a permutation $\sigma k$ of $\{1, \ldots, N\}$ with

$$
\operatorname{Re}\left(t\left\langle\Lambda, a^{\sigma k(1)}\right\rangle\right) \leq \operatorname{Re}\left(t\left\langle\Lambda, a^{\sigma k(2)}\right\rangle\right) \leq \ldots \leq \operatorname{Re}\left(t\left\langle\Lambda, a^{\sigma k(N)}\right\rangle\right) \quad\left(t \in S_{k}\right) .
$$

(iii) The sectors $S_{k}$ are the maximal sectors (3.3) with the properties (i) and (ii). We note that the permutations $\sigma k$ are in general not yet uniquely determined; this is not necessary. In addition here we can assume for convenience that $\sigma 1=\mathrm{id}$.

From the results on the asymptotic behaviour of the zeros of exponential sums [16] (see also [4: Satz 2.1.1] for further details) and from formula (2.10) we infer in the next lemma that $\lim _{r \rightarrow \infty} W\left(r e^{i \varphi} ; W_{C}\right)$ exists for $\varphi \neq \varphi_{j}$.

Lemma 2. Let $\varepsilon>0$ and let $C \in \boldsymbol{C}^{(n+m) \times n}$ be such that $Q_{0}(C)$ is regular. Then almost all zeros (i.e. except at most finitely many) of det $Q(t)$ - defined by (2.4) - are contained in the set

$$
\bigcup_{k=1}^{2 h}\left\{t \in \boldsymbol{C} \mid \varphi_{k}-\varepsilon<\arg t<\varphi_{k}+\varepsilon\right\} \text {. }
$$

In addition, for $\varphi \in[0,2 \pi] \backslash\left\{\varphi_{1}, \ldots, \varphi_{2 h}\right\}, 1 \leq \ell \leq m$ and $1 \leq \alpha \leq n$ there exists

$$
w_{\ell \alpha}\left(e^{i \varphi} \cdot \infty ; W_{C}\right):=\lim _{r \rightarrow \infty} w_{\ell \alpha}\left(r e^{i \varphi} ; W_{C}\right)
$$

with respect to the chordal metric on the Riemann sphere $\widehat{\mathbf{C}}=\mathbf{C} \cup\{\infty\}$. The limits are easily determined from formula (2.10).

3.3 Three representations of $\Gamma$. There are several possibilities to determine the set $\Gamma$ of all (complex) solutions of equation (ARE):

(i) $\Gamma=\left\{P Q^{-1} \mid\left(\begin{array}{l}Q \\ P\end{array}\right) \in \boldsymbol{C}^{(n+m) \times n}, \operatorname{det} Q \neq 0, \operatorname{span}\left(\begin{array}{l}Q \\ P\end{array}\right)\right.$ is $B-$ invariant $\}$ (see [19: Theorem 1] for an alternative formulation and a proof).

(ii) $\Gamma=\boldsymbol{C}^{m \times n} \cap\left\{\dot{W}\left(e^{i \varphi} \cdot \infty ; W_{C}\right) \mid C \in \boldsymbol{C}^{(n+m) \times n}, Q_{0}(C)\right.$ regular $\}$ for $\varphi \notin$ $\left\{\varphi_{1}, \varphi_{2}, \ldots, \varphi_{2 h}\right\}$. This is an immediate consequence of Lemma 2 and formula(2.10).

(iii) $\Gamma=\left\{\right.$ all constant $C^{m \times n}$-valued functions $W\left(\cdot ; W_{C}\right)$ of the form (2.10) $\}$.

Using (i) - (iii) and the fundamental representation formula (2.10) we obtain a nice parametrization of $\Gamma$. For this purpose we introduce some abbreviations. 
3.4 Notations. Let $V_{n}\left(\boldsymbol{C}^{n+m}\right)$ be the set of all full rank $(n+m) \times n$ matrices with complex entries. For $C \in V_{n}\left(\boldsymbol{C}^{n+m}\right)$ and $1 \leq k \leq N$ we set

$$
\begin{aligned}
J_{0}(C) & =\left\{j \mid 1 \leq j \leq N \text { and } D\left(a^{j}, C\right) \neq 0\right\} \\
G_{\zeta}\left(a^{k}\right) & =\left\{C \in V_{n}\left(\boldsymbol{C}^{n+m}\right) \mid D\left(a^{\nu}, C\right)=0 \text { for } \zeta\left(a^{\nu}\right) \neq \zeta\left(a^{k}\right) \text { and } D\left(a^{k} ; C\right) \neq 0\right\}
\end{aligned}
$$

and, for $1 \leq \ell \leq m$ and $1 \leq \alpha \leq n$,

$$
\begin{aligned}
\operatorname{nom}_{\ell \alpha}(C) & =\sum_{\nu \in J_{0}(C)} p_{\ell \alpha}\left(t, a^{\nu}\right) D\left(a^{\nu}, C\right) \\
\operatorname{den}(C) & =\sum_{\nu \in J_{0}(C)} p\left(t, a^{\nu}\right) D\left(a^{\nu}, C\right) \\
d_{n}(C) & =\max \left\{\operatorname{degree}\left(\operatorname{nom}_{\ell \alpha}(C)\right) \mid 1 \leq \ell \leq m, 1 \leq \alpha \leq n\right\} \\
d_{d}(C) & =\operatorname{degree}(\operatorname{den}(C))
\end{aligned}
$$

where here and in the sequel - for technical reasons - the degree of the zero polynomial is defined to be $-\infty$.

Notice that for $C \in G_{\zeta}\left(a^{k}\right)$ all exponential terms in (3.2) are identical to $e^{t \zeta\left(a^{k}\right)}$ Hence in this case

$$
w_{\ell \alpha}\left(t ; W_{C}\right)=\frac{\operatorname{nom}_{\ell \alpha}(C)}{\operatorname{den}(C)} \quad \text { if } \quad d_{d}(C) \geq 0
$$

In particular $W\left(t ; W_{C}\right)$ is a constant solution if $d_{d}(C)=0$ and $d_{n}(C) \leq 0$.

For the description of $\Gamma$ we use the set of parameter matrices

$$
G_{\zeta}^{0}\left(a^{k}\right)=\left\{C \in G_{\zeta}\left(a^{k}\right) \mid d_{d}(C)=0 \text { and } d_{n}(C) \leq 0\right\}
$$

and the set of indices

$$
J\left(a^{k}\right)=\bigcup_{C \in G_{\zeta}^{0}\left(a^{k}\right)} J_{0}(C)
$$

3.5 Parametrization of $\Gamma$. a) To any matrix $C \in G_{\zeta}^{0}\left(a^{k}\right) \neq 0$ there corresponds according to formula (2.10) - a solution

$$
W_{C} \equiv W\left(t ; W_{C}\right)=:\left(w_{\ell \alpha}^{C}\right)_{\substack{1 \leq \ell \leq m \\ 1 \leq \alpha \leq n}}
$$

of equation (ARE), where

$$
w_{\ell \alpha}^{C}=\frac{\sum_{\nu \in J_{0}(C)} p_{\ell \alpha}\left(t, a^{\nu}\right) D\left(a^{\nu}, C\right)}{\sum_{\nu \in J_{0}(C)} p\left(t, a^{\nu}\right) D\left(a^{\nu}, C\right)} .
$$


On the other hand it follows from Subsection $3.3 /(\mathrm{i})$, (iii) and formula (3.2) that any solutions of equation (ARE) can be represented in the form (3.5) (see also Theorem $3 /(i))$.

To each family

$$
F_{k}=\left\{C \in V_{n}\left(\boldsymbol{C}^{n+m}\right) \mid J_{0}(C) \subset J\left(a^{k}\right)\right\}
$$

there corresponds the family $\Gamma_{k}=\left\{W_{C} \mid C \in F_{k}\right\}$ of solutions of equation (ARE) and $\Gamma=\bigcup_{k=1}^{N} \Gamma_{k}$.

It is well known that the number of solutions of equation (ARE) corresponds to the number of $n$-dimensional $B$-invariant subspaces of $\boldsymbol{C}^{n+m}$ being complementary to the span of $\left(\begin{array}{c}0_{n, m} \\ I_{m}\end{array}\right)$ (see Subsection $3.3 /(\mathrm{i})$ ). Alternatively the number of elements of $\Gamma$ and the structure of $\Gamma$ can be easily determined from (3.5) if we know \# $J_{0}(C)$ and $\# J\left(a^{k}\right)(1 \leq k \leq N)$. Obviously $\Gamma$ is either finite or uncountable.

The following Lemma implies in particular that $\Gamma_{k} \cap \Gamma_{j}=\emptyset$ for $k \neq j$ if $B$ has only simple eigenvalues.

Lemma 3. Let $C \in G_{\zeta}\left(a^{j}\right)$ and $D\left(a^{\alpha}, C\right) \neq 0$. Then

$$
\left(\lambda_{\nu_{1}^{j}}, \cdots, \lambda_{\nu_{n}^{j}}\right)=\left(\lambda_{\nu_{1}^{\alpha}}, \cdots, \lambda_{\nu_{n}^{\alpha}}\right) .
$$

Proof. From the definition of $G_{\zeta}\left(a^{j}\right)$ it follows that $\zeta\left(a^{j}\right)=\zeta\left(a^{\alpha}\right)$. Let $\alpha \neq j$ and let us assume that the assertion of Lemma 3 does not hold. Then there is a $q$ with $\lambda_{\nu_{i}^{a}}=$ $\lambda_{\nu_{i}^{j}}$ for $q+1 \leq i \leq n$ and (without loss of generality) $\operatorname{Re}\left(\lambda_{\nu_{q}} e^{i \varphi}\right)<\operatorname{Re}\left(\lambda_{\nu_{q}^{j}} e^{i \varphi}\right)$ for $0<$ $\varphi<\varepsilon_{0}$. Since $c_{\nu_{1}^{j}}, \ldots, c_{\nu_{n}^{j}}$ are linearly independent, we have $c_{\nu_{q}^{\alpha}}=\sum_{i=1}^{n} \gamma_{i} c_{\nu_{i}^{j}}$ and from $\left|c_{\nu_{1}^{\mathrm{o}}} \cdots c_{\nu_{n}^{\mathrm{a}}}\right|^{\top} \neq 0$ it follows that $\left(\gamma_{1}, \ldots, \gamma_{q}\right) \neq 0$ since $c_{\nu_{q}^{\mathrm{a}}}, c_{\nu_{q+1}^{\mathrm{o}}}=c_{\nu_{q+1}^{j}}, \ldots, c_{\nu_{n}^{\mathrm{o}}}=c_{\nu_{n}^{j}}$ are linearly independent.

Let $\gamma_{p} \neq 0$ for some $p \in\{1, \ldots, q\}$. Then

$$
\left|c_{\nu_{1}^{j}} \cdots c_{\nu_{p-1}^{j}} c_{\nu_{q}^{\alpha}} c_{\nu_{p+1}^{j}} \cdots c_{\nu_{n}^{j}}\right|^{\top}=\gamma_{p}\left|c_{\nu_{1}^{j}} \cdots c_{\nu_{n}^{j}}\right|^{\top} \neq 0
$$

and

$$
\operatorname{Re}\left(e^{i \varphi}\left[\lambda_{\nu_{1}^{j}}+\ldots+\lambda_{\nu_{p-1}^{j}}+\lambda_{\nu_{q}^{a}}+\lambda_{\nu_{p+1}^{j}}+\ldots+\lambda_{\nu_{n}^{j}}\right]\right)<\operatorname{Re}\left(e^{i \varphi} \zeta\left(a^{j}\right)\right)
$$

for $0<\varphi<\varepsilon_{0}$. This contradicts $C \in G_{\zeta}\left(a^{j}\right)$ and Lemma 3 is proved

b) From the definition of $J\left(a^{k}\right)$ we infer using Lemmas 1 and 3 that $\# J\left(a^{k}\right) \leq 1$ if at most one of the eigenvalues $\lambda_{\nu_{1}^{k}}, \ldots, \lambda_{\nu_{n}^{k}}$ is a multiple eigenvalue and if this eigenvalue has geometric multiplicity one.

If $\# J\left(a^{k}\right)=0$, then $\Gamma_{k}=0$ and if $\# J\left(a^{k}\right)=1$, then $\Gamma_{k}=\left\{W_{k}\right\}$ where $W_{k}=$ $\left(w_{k, \ell \mu}\right)_{\substack{1 \leq \ell \leq m \\ 1 \leq \mu \leq n}}$ with

$$
w_{k, \ell \mu}=\frac{p_{\ell \mu}\left(t, a^{k}\right)}{p\left(t, a^{k}\right)}=\frac{\left|v_{\nu_{1}^{k}}(\ell, \mu), \ldots, v_{\nu_{n}^{k}}(\ell, \mu)\right|}{\left|\tilde{v}_{\nu_{1}^{k}}, \ldots, \tilde{v}_{\nu_{n}^{k}}\right|} .
$$

If $\# J\left(a^{k}\right)=\kappa>1$, then $\Gamma_{k}$ is uncountable and there are several possibilities for the structure of $\Gamma_{k}$. 
If there are matrices $C \in G_{\zeta}^{0}\left(a^{k}\right)$ with $J_{0}(C)=J\left(a^{k}\right)$, then we infer from formula (3.2) that $\Gamma_{k}$ is a $(\kappa-1)$-parametric family of solutions of equation (ARE) of the form (3.5) - in this case we can assume without loss of generality that $D\left(a^{k}, C\right)=1$ if this parameter is non-zero.

Otherwise, according to Lemma 3 and formula (3.2), we can split $\Gamma_{k}$ into two or more subsets:

$$
\Gamma_{k}=\bigcup_{j=1}^{n_{k}} \Gamma_{k_{j}} \text { where } \Gamma_{k_{j}} \text { is a } p_{k_{j}} \text { - parametric set and } \sum_{j=1}^{n_{k}} p_{k_{j}} \leq \kappa-1
$$

Since every element of $\Gamma$ can be written in the form (3.5) (for some $k$ ) we have obtained a parametrization of all complex solutions of equation (ARE) (see also Theorem 3 ).

A detailed description of all real symmetric solutions of equation (ARE) has been given by Shayman $[24,25]$, the set of all complex solutions of equation (ARE) has been determined by Meyer [19] (see Subsection 3.3/(i)).

c) If the matrix $B$ has $n+m$ simple eigenvalues, then, according to item b), equation (ARE) has at most $N$ different solutions. This fact has already been observed by Potter [20] and Mårtensson [17: p. 26], who considered the most important special case of equations (RDE) and (ARE).

If to each eigenvalue of $B$ there corresponds exactly one Jordan block (which means that the numbers $\mu_{\nu}$, defined at the beginning of Section 2, are pairwise different), then we get similarly that equation (ARE) has only a finite number $N_{0} \leq N$ of solutions which can be determined according to item b). In addition $N_{0}$ can be determined from the number of the Jordan blocks of $B$ and from its eigenvectors of rank lesser or equal $n$.

If $B$ is cyclic, which means that $J=V^{-1} B V$ consists of exactly one Jordan block, then equation (ARE) has no solution if $D=\left|\tilde{v}_{1}, \ldots, \tilde{v}_{n}\right|=0$, otherwise equation (ARE) has exactly one solution $W_{0}=\left(w_{\ell \alpha}^{0}\right)$ with

$$
w_{\ell \alpha}^{0}=\frac{1}{D}\left|v_{1}(\ell, \alpha), \ldots, v_{n}(\ell, \alpha)\right|
$$

This corresponds to [19: Corollary 1].

d) For special cases of equation (ARE) there are control-theoretical conditions, ensuring that the denominators of $(3.2),(3.5)$ or (3.6) are non-zero (see $[15,17,18,23$ $-27,28])$. For a survey of the most important results on the symmetric and definite solutions of equation (ARE) in the special case $B=\left(\begin{array}{cc}A & B_{0} \\ C & A^{T}\end{array}\right)$ see [15].

3.6 The parameter matrices $C$ and $W_{C}$. In order to parametrize the solution $W\left(\cdot ; W_{C}\right)$ it is convenient to use the matrix $C$ instead of the initial matrix $W_{C}$. Here we describe how $C$ can be determined from the initial matrix.

Let $C \in V_{n}\left(\mathbf{C}^{n+m}\right)$ and $W_{C}=P_{0}(C) Q_{0}(C)^{-1}$ (see (2.4)). If $K \in \boldsymbol{C}^{n \times n}$ is regular and $\widetilde{C}=C K$, then $W_{\widetilde{C}}=W_{C}$. Hence to the class

$$
[C]=\{C K \mid K \in G l(n, \boldsymbol{C})\} \quad \text { with } \operatorname{det} Q_{0}(C) \neq 0
$$


there corresponds a unique matrix $W_{C}$.

Let $W_{C_{0}}$ be given. Then we can determine a normalized representative $C \in\left[C_{0}\right]$ in the following way:

Version 1: Set $C=V^{-1}\left(\begin{array}{c}I_{n} \\ W_{C_{0}}\end{array}\right)$.

Version 2: (i) Choose a permutation $\pi$ of $\{1, \ldots, n+m\}$ and define

$$
V_{11} \in \boldsymbol{C}^{n \times m}, \quad V_{12} \in \boldsymbol{C}^{n \times n}, \quad V_{21} \in \boldsymbol{C}^{m \times m}, \quad V_{22} \in \boldsymbol{C}^{m \times n}
$$

by

$$
V_{\pi}=\left(v_{\pi(1)}, \ldots, v_{\pi(n+m)}\right)=\left(\begin{array}{ll}
V_{11} & V_{12} \\
V_{21} & V_{22}
\end{array}\right) .
$$

(ii) Check if the system of equations

$$
\left(W_{C_{0}} V_{11}-V_{21}\right) \widehat{C}=V_{22}-W_{C_{0}} V_{12}
$$

for $\widehat{C}=:\left(c_{\pi(1)} \cdots c_{\pi(m)}\right)^{\top} \in \boldsymbol{C}^{m \times n}$ is solvable.

If a solution $\widehat{C}$ of equation (3.7) exists with $\operatorname{det}\left(V_{11} \widehat{C}+V_{12}\right) \neq 0$, then we set $c_{\pi(m+j)}=e_{j} \quad(1 \leq j \leq n)$ with the canonical unit vectors $e_{j} \in \boldsymbol{C}^{1 \times n}$ and we set $C=\left(c_{1} \cdots c_{n+m}\right)^{\top}$. In this case it follows from $\widehat{V}\left(\begin{array}{l}\widehat{C} \\ I_{n}\end{array}\right)=V C$ and equation (3.7) that

$$
W_{C_{0}}=\left(V_{21} \widehat{C}+V_{22}\right)\left(V_{11} \hat{C}+V_{12}\right)^{-1}=W_{C}
$$

If $C$ cannot be determined using $\pi$, then we choose succesively another of the $N$ permutations of $\{1, \ldots, n+m\}$ until - after at most $N$ steps - $C$ has been determined. We propose to start in step (i) with $\pi=$ id since in most applications one is mainly interested in the case where equation (3.7) is solvable for $\pi=\mathrm{id}$ (see Remark $3 /(\mathrm{ii})$ ). Notice that the matrix $\widehat{C}$ is of lower dimension than the matrix $C$ in Version 1 , hence from computational point of view it is often better to use Version 2 instead of Version 1.

\section{The phase portrait of equations $(\mathrm{RDE})_{\mathbb{R}}$ and $(\mathrm{RDE})$}

Let $C \in V_{n}\left(\boldsymbol{C}^{n+m}\right)$ be such that $Q_{0}(C)$ is regular, $\varepsilon>0$ and $\varphi \in\left\{\varphi_{1}, \ldots, \varphi_{2 h}\right\}$. Then $W\left(\cdot ; W_{C}\right)$ can have an infinite number of poles in the sector $\{t \in \boldsymbol{C} \mid \varphi-\varepsilon<\arg t<\varphi+\varepsilon\}$ and - in contrast to $(3.4)-\lim _{r \rightarrow \infty} w_{\ell \alpha}\left(r e^{i \varphi} ; W_{C}\right)$ may fail to exist. According to Lemma 2 the phase portrait of equation (RDE) is (as $t \rightarrow \infty$ ) very simple if we restrict $t$ to the interior of a sector $S_{k} \quad(1 \leq k \leq 2 h)$. On the other hand the behaviour of $W\left(\cdot ; W_{C}\right)$ along one of the rays $B_{\varphi}=\{t \in \boldsymbol{C} \mid \arg t=\varphi\}$ may be very complicated. Obviously it is sufficient to describe $W\left(\cdot ; W_{C}\right)$ along one of the rays $B_{\varphi_{j}} \quad(1 \leq j \leq 2 h)$ - without loss of generality we assume that the positive (and consequently also the negative) half-axis is one of these rays. For this reason we confine in this section to the description of the phase portrait of equation $(\mathrm{RDE})_{\mathbb{R}}$. 
For the rest of this section let $k \in\{1, \ldots, N\}, C \in V_{n}\left(\boldsymbol{C}^{n+m}\right)$ and $t \in \mathbb{R}$. The formulation of the following theorems is rather technical since we are considering the most general case - the formulations become much simpler if $B$ has simple eigenvalues or even if $B$ is only diagonalizable. For a detailed discussion of the fundamental representation formula we use the following abbreviations:

$$
\begin{aligned}
& d\left(a^{k}, C\right)=\operatorname{degree}\left(\sum_{\zeta\left(a^{j}\right)=\zeta\left(a^{k}\right)} p\left(t, a^{j}\right) D\left(a^{j}, C\right)\right) \\
& d_{\ell \alpha}\left(a^{k}, C\right)=\operatorname{degree}\left(\sum_{\zeta\left(a^{j}\right)=\zeta\left(a^{k}\right)} p_{\ell \alpha}\left(t, a^{j}\right) D\left(a^{j}, C\right)\right) \quad\left(\begin{array}{l}
1 \leq \ell \leq m \\
1 \leq \alpha \leq n
\end{array}\right) \\
& \widetilde{G}_{\zeta}\left(a^{k}\right)=\left\{\begin{array}{l|l}
C \in V_{n}\left(\boldsymbol{C}^{n+m}\right) & \begin{array}{l}
D\left(a^{k}, C\right) \neq 0, \text { and } D\left(a^{j}, C\right)=0 \\
\text { if } R\left(a^{j}\right)>R\left(a^{k}\right) \text { or } R\left(a^{j}\right)=R\left(a^{k}\right) \\
\text { as well as } \zeta\left(a^{j}\right) \neq \zeta\left(a^{k}\right)
\end{array}
\end{array}\right\} \\
& \widetilde{G}_{\zeta}^{0}\left(a^{k}\right)=\left\{\begin{array}{l|l}
C \in \widetilde{G}_{\zeta}\left(a^{k}\right) & \begin{array}{l}
d\left(a^{k}, C\right)=0 \text { and } \\
\max _{\ell, \alpha} d_{\ell \alpha}\left(a^{j}, C\right) \leq 0 \text { for } R\left(a^{j}\right)=R\left(a^{k}\right)
\end{array}
\end{array}\right\} .
\end{aligned}
$$

We shall see that each solution $W\left(\cdot ; W_{C}\right)$ of equation ??? with $C \in \widetilde{G}_{\zeta}^{0}\left(a^{k}\right)$ has a limit $W_{C_{0}} \in \Gamma$ with $C_{0} \in G_{\zeta}^{0}\left(a^{k}\right)$ as $t \rightarrow \infty$.

The following theorem is concerned with an important special case.

Theorem 2. Let $C \in G_{\zeta}^{0}\left(a^{k}\right)(\neq \emptyset)$ and $R\left(a^{j}\right) \neq R\left(a^{k}\right)$ for $j \neq k$. Then the following statements are true.

(i) $W_{C}:=W_{k}$ with $W_{k}$ defined by (3.6) is a solution of equation (ARE); $W_{k}$ is real if $B$ is real.

(ii) If $C^{1} \in \tilde{G}_{\zeta}^{0}\left(a^{k}\right)$ with $\operatorname{det} Q_{0}\left(C^{1}\right) \neq 0$, then there exist $t_{0} \in \mathbb{R}, \nu_{0} \in \mathbb{N}_{0}$ and $K_{0}>0$ such that $W\left(t ; W_{C^{1}}\right) \in C^{m \times n}$ for $t \geq t_{0}$ and

$$
\left|w_{\ell \alpha}\left(t, W_{C^{1}}\right)-\frac{\left|v_{\nu_{1}^{k}}(\ell, \alpha), \ldots, v_{\nu_{n}^{k}}(\ell, \alpha)\right|}{\left|\tilde{v}_{\nu_{1}^{k}}, \ldots, \tilde{v}_{\nu_{n}^{k}}\right|}\right| \leq K_{0} t^{\nu_{0}} e^{t\left(R\left(a^{k-1}\right)-R\left(a^{k}\right)\right)}
$$

for $1 \leq \ell \leq m, 1 \leq \alpha \leq n$ and $t \geq t_{0}$. This means that the convergence of $W\left(t, W_{C^{1}}\right)$ for $t \rightarrow \infty$ takes place at an exponential rate. We have $\nu_{0}=0$ if $B$ is semisimple, otherwise $0 \leq \nu_{0} \leq \max \left\{\operatorname{degree}\left(p_{\ell_{\mu}}\left(t, a^{j}\right)\right) \mid 1 \leq \mu \leq n, 1 \leq \ell \leq m, j \in J_{0}\left(C_{1}\right)\right\}$.

(iii) If $C^{2} \in \widetilde{G}_{\zeta}\left(a^{k}\right)$ with $d\left(a^{k}, C^{2}\right)=-\infty<\max _{\ell, \alpha} d_{\ell \alpha}\left(a^{k}, C^{2}\right)$, then either $\operatorname{det} Q_{0}\left(C^{2}\right)=0$ and $W_{C^{2}}$ is undefined or at least one element of $W\left(t, W_{C^{2}}\right)$ tends to infinity for $t \rightarrow \infty$.

Proof. Since $R\left(a^{j}\right) \neq R\left(a^{k}\right)$ for $j \neq k$, the eigenvalues $\lambda_{\nu_{1}^{k}}, \ldots, \lambda_{\nu_{n}^{k}}$ and the corresponding eigenvectors appear in conjugate complex pairs if $B$ is real. Hence it follows easily (as with [17: Theorem 3]) that $W_{k}$ is real if $B$ is real. 
From the assumptions of (ii) and from (3.2) we infer

$$
w_{\ell \alpha}\left(t ; W_{C^{1}}\right)=\frac{\sum_{j \in J_{0}\left(C^{1}\right)} e^{\ell \zeta\left(a^{j}\right)} p_{\ell \alpha}\left(t, a^{j}\right) D\left(a^{j}, C^{1}\right)}{\sum_{j \in J_{0}\left(C^{1}\right)} e^{\ell \zeta\left(a^{j}\right)} p\left(t, a^{j}\right) D\left(a^{j}, C^{1}\right)}
$$

where $p\left(t, a^{k}\right) D\left(a^{k}, C^{1}\right) \neq 0$ and $R\left(a^{j}\right)<R\left(a^{k}\right)$ for $j \in J_{0}\left(C^{1}\right) \backslash\{k\}$. Using (4.2) we obtain assertion (ii) of the theorem, and in the special case $C^{1}=C$ obviously $W_{C^{\mathrm{i}}}=W_{C}=W_{k}$.

Assertion (iii) is an immediate consequence of formula (3.2)

The next theorem generalizes Theorem 2.

Theorem 3. The following statements are true.

(i) If $C \in G_{\zeta}^{0}\left(a^{k}\right)$, then $W_{C}=:\left(w_{\ell \alpha}\right)_{\substack{1 \leq \ell \leq m \\ 1 \leq \alpha \leq n}}$ is a solution of equation (ARE) with

$$
\begin{gathered}
w_{\ell \alpha}=\frac{\sum_{j \in J_{0}(C)} p_{\ell \alpha}\left(t, a^{j}\right) D\left(a^{j}, C\right)}{\sum_{j \in J_{0}(C)} p\left(t, a^{j}\right) D\left(a^{j}, C\right)} \\
w_{\ell \alpha}=\frac{\sum_{j \in J_{0}(C)}\left|v_{\nu_{1}^{j}}(\ell, \alpha), \cdots, v_{\nu_{n}^{j}}(\ell, \alpha)\right|\left|\begin{array}{c}
c_{\nu_{1}^{j}} \\
\vdots \\
c_{\nu_{n}^{j}}
\end{array}\right|}{\sum_{j \in J_{0}(C)}\left|\tilde{v}_{\nu_{1}^{j}}, \cdots, \tilde{v}_{\nu_{n}^{j}}\right|\left|\begin{array}{c}
c_{\nu_{1}^{j}} \\
\vdots \\
c_{\nu_{n}^{j}}
\end{array}\right|}
\end{gathered}
$$

Notice that all polynomials $p_{\ell \alpha}\left(\cdot, a^{j}\right)$ and $p\left(\cdot, a^{j}\right)$ in (4.3) are constant. Any solution of equation (ARE) can be written in the form (4.4) for an adequate $C \in G_{\zeta}^{0}\left(a^{k}\right)$.

(ii) Let $C^{0} \in \widetilde{G}_{\zeta}^{0}\left(a^{k}\right)$. Then there exists a solution $W_{0}$ of equation (ARE) with $\lim _{t \rightarrow \infty} W\left(t ; W_{C^{0}}\right)=W_{0}$, and the convergence takes place at an exponential rate. $W_{0}$ is obtained from the right-hand side of (4.3) by replacing therein $C$ by $C^{0}$ and $j \in J_{0}(C)$ by $R\left(a^{j}\right)=R\left(a^{k}\right)$.

(iii) Let $C^{1} \in \tilde{G}_{\zeta}\left(a^{k}\right)$,

$$
\begin{aligned}
\Delta(t) & =\sum_{R\left(a^{j}\right)=R\left(a^{k}\right)} p\left(t, a^{j}\right) D\left(a^{j}, C^{1}\right) \\
\Delta \ell \alpha(t) & =\sum_{R\left(a^{j}\right)=R\left(a^{k}\right)} p_{\ell \alpha}\left(t, a^{j}\right) D\left(a^{j}, C^{1}\right) .
\end{aligned}
$$

If $-\infty<d=$ degree $\Delta(t) \geq \max \left\{\right.$ degree $\left.\Delta_{\ell \alpha}(t) \mid 1 \leq \ell \leq m, 1 \leq \alpha \leq n\right\}=D$, then there exists a solution $W_{0}$ of equation (ARE) with

$$
\lim _{t \rightarrow \infty} W\left(t ; W_{C^{1}}\right)=W_{0}
$$


and $W_{0}$ can be determined from (3.2) and the coefficients of $t^{d}$ in the polynomials $\Delta(t)$ and $\Delta_{\ell \alpha}(t)$. In addition there exist constants $k>0$ and $t_{0}>0$ with

$$
\left|w_{\ell \alpha}\left(t ; W_{C^{1}}\right)-w_{\ell \alpha}\left(t ; W_{0}\right)\right| \leq \frac{k}{t} \quad \text { for } t \geq t_{0} \quad \text { and } \quad 1 \leq \ell \leq m, 1 \leq \alpha \leq n
$$

In this case we say that the convergence takes place at a polynomial rate.

If $0 \leq d<D$, then at least one element $w_{\ell a}\left(t ; W_{C^{1}}\right)$ tends to $\infty$ as $x \rightarrow \infty$ and if $\Delta(t) \equiv 0$, then the limit in (4.5) may fail to exist.

Proof. Assertion (i) follows from Subsection 3.5 - the last sentence in assertion (i) is a consequence of Subsection 3.3/(i) and (2.10). Notice that (4.4) results from (4.3) and the definition of $G_{\zeta}^{0}\left(a^{k}\right)$.

From the definition of $\widetilde{G}_{\zeta}^{0}$ and (3.2) we infer that

$$
w_{\ell \alpha}\left(t ; W_{C^{0}}\right)=\frac{\sum_{R\left(a^{j}\right) \leq R\left(a^{k}\right)} e^{\ell \zeta\left(a^{j}\right)} p_{\ell \alpha}\left(t, a^{j}\right) D\left(a^{j}, C^{0}\right)}{\sum_{R\left(a^{j}\right) \leq R\left(a^{k}\right)} e^{t \zeta\left(a^{j}\right)} p\left(t, a^{j}\right) D\left(a^{j}, C^{0}\right)}
$$

where

$$
\sum_{R\left(a^{j}\right)=R\left(a^{k}\right)} p\left(t, a^{j}\right) D\left(a^{j}, C^{0}\right)
$$

is a non-zero constant. Obviously this implies assertion (ii). Part (iii) of the theorem is proved in the same way

Remark 2. (i) Since any initial value problem for equation (RDE) is solvable and since the solution can be represented in the form (2.10), it follows from (2.10) (for $t=0$ ) that for an arbitrary initial matrix $W_{0} \in \boldsymbol{C}^{m \times n}$ we can choose $k \in\{1, \ldots, N\}$ such that there exists a matrix $C \in G_{\zeta}\left(a^{k}\right)$ with $W_{0}=W_{C}$ such that at least one of the constants $d$ and $D$ defined in Theorem $3 /$ (iii) is unequal $-\infty$.

(ii) Using item (i), formula (2.10) and Theorem 3 we can determine the stable set $S\left(W_{C}\right)$ of an arbitrary solution $W_{0}=W_{C}$ of equation (ARE). If $C \in G_{\zeta}\left(a^{k}\right)$ and if $B$ is semisimple, then it follows from formula $(2.10)$ and Theorem 3 that $S\left(W_{C}\right)=$ $\left\{W_{C_{0}} \mid C_{0} \in \widetilde{G}_{\zeta}^{0}\left(a^{k}\right)\right\}$.

If $B$ is not semisimple, then the situation is more involved (see Theorem $3 /(\mathrm{iii})$ ) - instead of trying to describe $S\left(W_{C}\right)$ in the general case we propose to use directly formula (2.10) to determine $S\left(W_{C}\right)$ for a fixed solution $W_{C}$ of equation (ARE).

(iii) The set of all (almost) periodic solutions of equation (RDE) $)_{\mathbb{R}}$ corresponds to the set of all (almost) periodic functions of the form (3.2). Using this fact and (3.2) we get the assertions of the two following theorems - the proofs are omitted since they are similar to the proofs of Theorems 1 and 2 . 
For the formulation of Theorem 4 we use the following abbreviations:

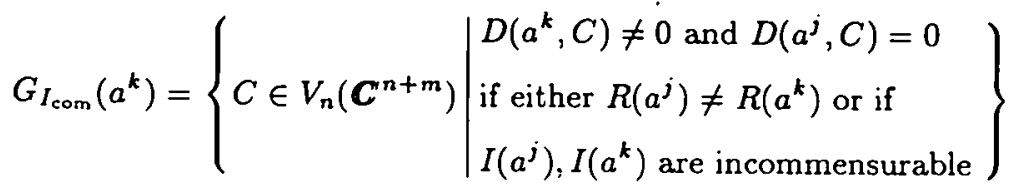

$$
\begin{aligned}
& G_{I_{\text {com }}}^{1}\left(a^{k}\right)=\left\{\begin{array}{l|l}
C \in G_{I_{\text {com }}}\left(a^{k}\right) & \begin{array}{l}
\text { if } R\left(a^{j}\right)=R\left(a^{k}\right), \text { then } \\
d\left(a^{j}, C\right) \leq 0 \text { and } \max _{\ell, \alpha} d_{\ell \alpha}\left(a^{j}, C\right) \leq 0
\end{array}
\end{array}\right\} \\
& \tilde{G}_{I_{\mathrm{com}}}\left(a^{k}\right)=\left\{\begin{array}{l|l}
C \in V_{n}\left(\boldsymbol{C}^{n+m}\right) & \begin{array}{l}
D\left(a^{k}, C\right) \neq 0, \text { and } D\left(a^{j}, C\right)=0 \text { if } \\
R\left(a^{j}\right)>R\left(a^{k}\right) \text { or } R\left(a^{j}\right)=R\left(a^{k}\right) \text { and } \\
I\left(a^{j}\right) \text { and } I\left(a^{k}\right) \text { are incommensurable }
\end{array}
\end{array}\right\} \\
& \widetilde{G}_{I_{\mathrm{com}}}^{1}\left(a^{k}\right)=\left\{\begin{array}{l|l}
C \in \widetilde{G}_{I_{\mathrm{com}}}\left(a^{k}\right) & \begin{array}{l}
\text { if } R\left(a^{j}\right)=R\left(a^{k}\right), \text { then } \\
d\left(a^{j}, C\right) \leq 0 \text { and } \max _{\ell, \alpha} d_{\ell \alpha}\left(a^{j}, C\right) \leq 0
\end{array}
\end{array}\right\} \\
& \tau_{I_{\text {com }}}\left(a^{k}\right)=\left\{j \mid R\left(a^{j}\right)=R\left(a^{k}\right), I\left(a^{j}\right) \text { and } I\left(a^{k}\right) \text { are commensurable }\right\} .
\end{aligned}
$$

Theorem 4. The following statements are true.

(i) Let $C \in G_{I_{\text {com }}}^{1}\left(a^{k}\right)$ with $\operatorname{det} Q_{0}(C) \neq 0$. Then $W\left(\cdot ; W_{C}\right)$ (restricted to $\left.\mathbb{R}\right)$ is a periodic solution of equation $(\mathrm{RDE})_{\mathbb{R}}$ with

$$
\begin{aligned}
& w_{\ell \alpha}\left(t ; W_{C}\right)=\frac{\sum_{j \in \tau_{I_{\text {com }}}\left(a^{k}\right)} e^{i t I\left(a^{j}\right)} p_{\ell \alpha}\left(t, a^{j}\right) D\left(a^{j}, C\right)}{\sum_{j \in \tau_{I_{\text {com }}}\left(a^{k}\right)} e^{i t I\left(a^{j}\right)} p\left(t, a^{j}\right) D\left(a^{j}, C\right)}
\end{aligned}
$$

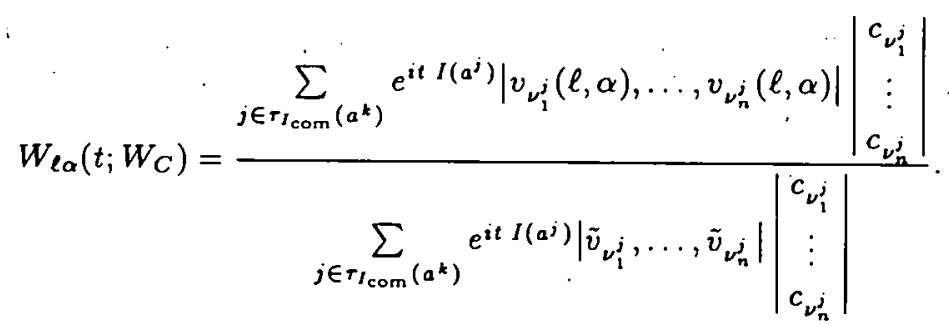

The solution $W\left(\cdot ; W_{C}\right)$ may have real poles. Every periodic solution of equation $(\mathrm{RDE})_{\mathbb{R}}$ is of the form (4.7) and (4.8) for some $k \in\{1, \ldots, N\}$ and for some matrix $C \in$ $G_{I_{\text {com }}}^{1}\left(a^{k}\right)$ or it can be written in the form (4.10) (see Theorem 5/(i)).

The set of all periodic solutions of equation $(\mathrm{RDE})_{\mathbb{R}}$ is either empty or uncountable.

(ii) Let

$$
C^{1}=\left(\begin{array}{c}
c_{1}^{1} \\
\vdots \\
c_{n+m}^{1}
\end{array}\right) \in \widetilde{G}_{I_{\mathrm{com}}}^{1}\left(a^{k}\right) \quad \text { and } \quad \widetilde{C}^{1}=\left(\begin{array}{c}
\tilde{c}_{1}^{1} \\
\vdots \\
\tilde{c}_{n+m}^{1}
\end{array}\right)
$$


with

$$
\tilde{c}_{k}^{1}= \begin{cases}c_{k}^{1} & \text { if } k \in \underset{j \in \tau_{I_{c o m}\left(a^{k}\right)}}{\bigcup}\left\{\nu_{1}^{j}, \ldots, \nu_{n}^{j}\right\} \\ 0 & \text { else }\end{cases}
$$

If

$$
\Delta_{0}(t)=\sum_{j \in \tau_{I_{c o m}\left(a^{k}\right)}} e^{t I\left(a^{j}\right)} p\left(t, a^{j}\right) D\left(a^{j}, \tilde{C}^{1}\right) \quad \text { and } \quad\left|\Delta_{0}(t)\right| \geq \delta>0 \quad(t \in \mathbb{R}),
$$

then $W\left(\cdot ; W_{\tilde{C}^{1}}\right)$ is a periodic solution of equation $(\mathrm{RDE})_{\mathbb{R}}$ with

$$
\lim _{t \rightarrow \infty}\left(W\left(t ; W_{C^{1}}\right)-W\left(t ; W_{\bar{C}^{1}}\right)\right)=0
$$

and the convergence takes place at an exponential rate.

(iii) Let $C^{2} \in \tilde{G}_{I_{\text {com }}}\left(a^{k}\right)$ and

$$
\sum_{R\left(a^{j}\right)=R\left(a^{k}\right)} e^{t \zeta\left(a^{j}\right)} p\left(t, a^{j}\right) D\left(a^{j}, C^{2}\right)=: e^{t \zeta\left(a^{j}\right)} \Delta(t)
$$

where

$$
\Delta(t)=\sum_{\zeta\left(a^{j}\right)=\zeta\left(a^{k}\right)} p\left(t, a^{j}\right) D\left(a^{j}, C^{2}\right)
$$

is a polynomial of degree $d>0$. If

$$
\max _{\ell, \alpha}\left(\operatorname{degree}\left(\sum_{\zeta\left(a^{\nu}\right)=\zeta\left(a^{j}\right)} p_{\ell \alpha}\left(t, a^{\nu}\right) D\left(a^{\nu}, C^{2}\right)\right)\right) \leq d \quad \text { for } R\left(a^{j}\right)=R\left(a^{k}\right),
$$

then there exists a periodic solution $W\left(\cdot ; W_{C}\right)$ of equation $(\mathrm{RDE})_{\mathbb{R}}$ with

$$
\lim _{t \rightarrow \infty}\left(W\left(t ; W_{C^{2}}\right)-W\left(t ; W_{C}\right)\right)=0
$$

and the convergence takes place at a polynomial rate. Using $(3.2), W\left(\cdot ; W_{C}\right)$ can be determined from the coefficients of $t^{d}$ in the polynomials $p\left(t, a^{j}\right)$ and $p_{\ell \alpha}\left(t, a^{j}\right) \quad(j \in$ $\left.\tau_{I_{\text {com }}}\left(a^{k}\right)\right)$.

For the formulation of Theorem 5 we set

$$
\begin{aligned}
& G_{R}\left(a^{k}\right)=\left\{\begin{array}{l|l}
C \in V_{n}\left(\boldsymbol{C}^{n+m}\right) & \begin{array}{l}
D\left(a^{k}, C\right) \neq 0 \text { and } \\
D\left(a^{j}, C\right)=0 \text { if } R\left(a^{j}\right) \neq R\left(a^{k}\right)
\end{array}
\end{array}\right\} \\
& G_{R}^{1}\left(a^{k}\right)=\left\{\begin{array}{l|l}
C \in G_{R}\left(a^{k}\right) & \begin{array}{l}
d\left(a^{j}, C\right) \leq 0 \text { and } \\
\max _{\ell, \alpha} d_{\ell \alpha}\left(a^{j}, C\right) \leq 0 \text { for } R\left(a^{j}\right)=R\left(a^{k}\right)
\end{array}
\end{array}\right\} \\
& \widetilde{G}_{R}\left(a^{k}\right)=\left\{\begin{array}{l|l}
C \in V_{n}\left(\boldsymbol{C}^{n+m}\right) & \begin{array}{l}
D\left(a^{k}, C\right) \neq 0 \text { and } \\
D\left(a^{j}, C\right)=0 \text { for } R\left(a^{j}\right)>R\left(a^{k}\right)
\end{array}
\end{array}\right\} \\
& \tilde{G}_{R}^{1}\left(a^{k}\right)=\left\{\begin{array}{l|l}
M \in \widetilde{G}_{R}\left(a^{k}\right) & \begin{array}{l}
d\left(a^{k}, C\right) \leq 0 \text { and } \\
d\left(a^{j}, C\right) \leq 0 \text { for } R\left(a^{j}\right)=R\left(a^{k}\right)
\end{array}
\end{array}\right\} .
\end{aligned}
$$


Theorem 5. The following statements are true.

(i) Let $C \in G_{R}^{1}\left(a^{k}\right)$. If $\operatorname{det} Q(t, C) \not \equiv 0$ and $\operatorname{det} Q_{0}(C) \neq 0$, then $W\left(\cdot ; W_{C}\right)$ is an almost periodic solution of equation $(R D E)_{\mathbb{R}}$ which is bounded if $\operatorname{det} Q(t ; C) \neq 0$ for $|\operatorname{Im} t| \leq \varepsilon>0$. The solution $W\left(\cdot ; W_{C}\right)$ satisfies

$$
\begin{aligned}
& w_{\ell \alpha}\left(t ; W_{C}\right)=\frac{\sum_{R\left(a^{j}\right)=R\left(a^{k}\right)} e^{t \zeta\left(a^{j}\right)} p_{\ell \alpha}\left(t, a^{j}\right) D\left(a^{j}, C\right)}{\sum_{R\left(a^{j}\right)=R\left(a^{k}\right)} e^{\ell \zeta\left(a^{j}\right)} p\left(t, a^{j}\right) D\left(a^{j}, C\right)}
\end{aligned}
$$

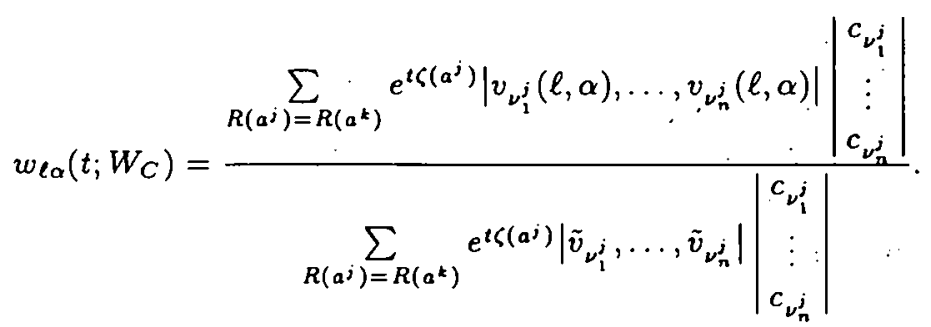

Every almost-periodic solution of equation (RDE) $)_{\mathbb{R}}$ is of these forms for some matrix $C \in G_{R}^{1}\left(a^{k}\right)$. Notice that the functions of the form (4.9) and (4.10) are periodic if the elements of the set $\left\{\zeta\left(a^{j}\right)-\zeta\left(a^{k}\right) \mid R\left(a^{j}\right)=R\left(a^{k}\right)\right\}$ are commensurable.

(ii) Let

$$
C^{1}=\left(\begin{array}{c}
c_{1}^{1} \\
\vdots \\
c_{n+m}^{1}
\end{array}\right) \in \tilde{G}_{R}^{1} \quad \text { and } \quad \tilde{C}^{1}=\left(\begin{array}{c}
\tilde{c}_{1}^{1} \\
\vdots \\
\tilde{c}_{n+m}^{1}
\end{array}\right)
$$

with

$$
\tilde{c}_{k}^{1}= \begin{cases}c_{k}^{1} & \text { if } k \in \bigcup_{R\left(a^{j}\right)=R\left(a^{k}\right)}\left\{\nu_{1}^{j}, \ldots, \nu_{n}^{j}\right\} \\ 0 & \text { else }\end{cases}
$$

If

$$
\Delta(t)=\sum_{R\left(a^{j}\right)=R\left(a^{k}\right)} e^{t \zeta\left(a^{j}\right)} p\left(t, a^{j}\right) D\left(a^{j}, C^{1}\right)=e^{t \zeta\left(a^{k}\right)} \Delta_{0}
$$

where

$$
\Delta_{0}=\sum_{R\left(a^{j}\right)=R\left(a^{k}\right)} p\left(t, a^{j}\right) D\left(a^{j}, C^{1}\right) \quad \text { and } \quad\left|\Delta_{0}(t)\right| \geq \delta>0 \quad\left(t \geq t_{0}\right),
$$

then $W\left(\cdot ; W_{\dot{C}^{1}}\right)$ is an almost-periodic solution of equation $(\mathrm{RDE})_{\mathbb{R}}$ with

$$
\lim _{t \rightarrow \infty}\left(W\left(t ; W_{C^{1}}\right)-W\left(t ; W_{\bar{C}^{1}}\right)=0\right.
$$

and the convergence takes place at an exponential rate.

(iii) The statement of Theorem 4 remains true if we replace therein $\widetilde{G}_{I_{\mathrm{com}}}\left(a^{k}\right)$ and periodic by $\widetilde{G}_{R}\left(a^{k}\right)$ and almost-periodic, respectively. 
Remark 3. (i) In the preceeding theorems we have given a description of the most important parts of the phase portrait of equation (RDE) $\mathbb{R}$. These results show that the phase portrait of the equations $(\mathrm{RDE})_{\mathbb{R}}$ and $(\mathrm{RDE})$ is rather simple if $B$ has only simple eigenvalues. If $B$ is semisimple (diagonalizable), then all polynomials $p\left(t, a^{j}\right)$ and $p_{\ell \alpha}\left(t, a^{j}\right)$ in (3.2) are constant and in this case the constant, periodic and almost-periodic solutions of equation $(R D E)_{\mathbb{R}}$ are of the form (4.4), (4.8) and (4.10), respectively - in addition we obtain from (3.2) quite easily the corresponding stable (or unstable) sets. The phase portrait of the symplectic Riccati equation with simple or semisimple spectrum has been described in detail by Shayman [27].

In the general case the phase portrait of equation $(\mathrm{RDE})_{\mathbb{R}}$ may be very complicated. On the other hand, using (3.2), it is not difficult to analyze the phase portrait of equation $(\mathrm{RDE})_{\mathbb{R}}$ if the matrix $B$ is fixed and if its Jordan canonical form and its (generalized) eigenvectors are known. There remains only to discuss the behaviour of some specific exponential sums as described in Theorems $2-5$.

(ii) An important special case is obtained if

$$
\operatorname{Re} \lambda_{m}<\operatorname{Re} \lambda_{m+1} \quad \text { and } \quad\left(\begin{array}{l}
A \\
B
\end{array}\right)=\left(v_{m+1}, \ldots, v_{m+n}\right)
$$

with

$$
A=\left(\tilde{v}_{m+1}, \ldots, \tilde{v}_{m+n}\right) \quad \text { and } \quad \operatorname{det} A \neq 0 .
$$

Then $W^{*}=B A^{-1}$ is a solution of equation (ARE) which is called dichotomic solution (see [18: Definition 2]). According to Theorem 2 any solution $W\left(\cdot ; W_{C}\right)$ with

$$
C=\left(\begin{array}{c}
c_{1} \\
\vdots \\
c_{n+m}
\end{array}\right) \quad \text { and } \quad\left|\begin{array}{c}
c_{m+1} \\
\vdots \\
c_{m+n}
\end{array}\right| \neq 0
$$

converges at an exponential rate to the dichotomic solution as $t \rightarrow \infty$. Hence the domain of attraction of $W^{*}$ is open and dense in $C^{m \times n}$. If rank $A=n-1$, then it follows with Lemma $1 /$ a) that at least one element of $W\left(\cdot ; W_{C}\right)$ tends to infinity as $t \rightarrow \infty$ if $\left|c_{m+1} \cdots c_{m+n}\right|^{\top} \neq 0$.

In the case of the symplectic Riccati equation the dichotomity condition $\operatorname{Re} \lambda_{m}<$ $\operatorname{Re} \lambda_{m+1}$ is equivalent to the condition $\operatorname{Re} \lambda_{j} \neq 0$ for $1 \leq j \leq n+m(=2 n)$.

(iii) If $\operatorname{Re} \lambda_{m}=\operatorname{Re} \lambda_{m+1}$, then it follows from formula (2.10) and Theorems 2 - 5 that there is no stable equilibrium of equation (RDE) with respect to $t \rightarrow \infty$ and that there is no solution of equation (ARE) whose domain of attraction is open and dense (see also [1]).

(iv) For the asymptotic behaviour of the solutions of equation (RDE) $)_{\mathbb{R}}$ for $t \rightarrow-\infty$ we obtain similarly all the corresponding results.

(v) All the results of this section can be formulated in the language of Grassmannian manifolds used in [27] and [8]. 


\section{Non-autonomous Riccati equations}

In this section we give an application of the fundamental representation formula (2.10) to the investigation of non-autonomous Riccati equations (RDE). In order to simplify the representation of the results we confine to the most important special case.

5.1 Assumptions. Let the coefficients $B_{i j}$ in equation (RDE) be matrix polynomials in $t$ such that

$$
B(t)=\left(\begin{array}{ll}
B_{11} & B_{12} \\
B_{21} & B_{22}
\end{array}\right)(t)=t^{r-1} \sum_{k=0}^{r-1} A_{k} t^{-k}
$$

with $r \in \mathbb{N}$,

$$
A_{k} \in \boldsymbol{C}^{(m+n) \times(m+n)} \quad \text { for } 0 \leq k \leq r-1
$$

and where

$$
A_{0}=\left(\begin{array}{ll}
A_{11}^{0} & A_{12}^{0} \\
A_{21}^{0} & A_{22}^{0}
\end{array}\right)
$$

has $n+m$ simple eigenvalues $\lambda_{1}, \ldots, \lambda_{n+m}$. The eigenvector of $A_{0}$ corresponding to $\lambda_{k}$ is denoted by $v_{k}(1 \leq k \leq n+m)$.

5.2 Notations and preliminary results. (i) The algebraic Riccati equation (ARE)

$$
0=A_{21}^{0}+A_{22}^{0} W-W A_{11}^{0}-W A_{12}^{0} W
$$

is called the limiting algebraic Riccati equation corresponding to

$$
\dot{W}=B_{21}(t)+B_{22}(t) W-W \cdot B_{11}(t)-W B_{12}(t) W .
$$

(ii) Let $\delta>0$ be sufficiently small and let here $\left(\phi_{k}\right)_{k \in \mathbb{Z}}$ with $\cdots<\phi_{0} \leq 0<\phi_{1}<$ $\phi_{2} \ldots$ be the sequence of the Stokes' directions (for the definition see [13: ???]) of the differential equation

$$
\dot{y}=B(t) y \quad(t \in C) \text {. }
$$

Then we set

$$
N_{k}(\delta)=\left\{t \in \boldsymbol{C} \mid \phi_{k-1}+\delta \leq \arg t \leq \phi_{k+1}-\delta\right\} .
$$

From Assumption 5.1 and from [3: ???] and [13: ???] we infer that for any sector $N_{k}(\delta)$ there exist $n+m$ linearly independent vector solutions $\hat{y}_{\mu} \quad(1 \leq \mu \leq n+m)$ of the linear differential equation (5.4) of the form

$$
\hat{y}_{\mu}(t)=x_{\mu}(t) \exp \left\{\sum_{j=0}^{r-1} \alpha_{j}^{(\mu)} \frac{t^{r-j}}{r-j}\right\}=:\left(t^{\beta_{\mu}} v_{\mu}+t^{\beta_{\mu}-1} b_{\mu}(t)\right) e^{\alpha_{\mu}(t)}
$$

where

$$
\alpha_{0}^{(\mu)}=\lambda_{\mu}, \quad \beta_{\mu} \in \boldsymbol{C}, \quad \alpha_{j}^{(\mu)} \in \boldsymbol{C} \quad(1 \leq j \leq r-1 ; 1 \leq \mu \leq n+m)
$$


and where $b_{\mu}(t) \quad(1 \leq \mu \leq n+m)$ is uniformly bounded for $t \in N_{k}(\delta)$. Since we shall assume that $N_{k}(\delta)$ is fixed, we do not indicate the dependence of $\hat{y}_{\mu}$ on the sector by an additional index.

(iii) We use all notations and assumptions introduced in the Sections 2 and 3 with the two following slight modifications:

a) Instead of the sectors $S_{k}$ we consider for $\delta \geq 0$ the sectors $S_{k}(\delta)$, defined in the following way:

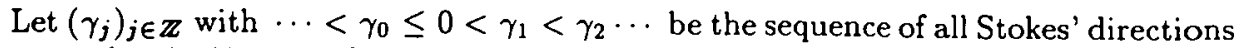
of the set $\left\{t^{r} \zeta\left(a^{\mu}\right) \mid a^{\mu} \in A\right\}$. Here, as in Subsection 3.1, $\zeta\left(a^{\mu}\right)=\lambda_{\nu_{1}^{\mu}}+\ldots+\lambda_{\nu_{n}^{\mu}}$. Then $\left\{\gamma_{j} \mid j \in \mathbb{Z}\right\}$ is the set of all solutions $\varphi$ of equations of the form

$$
f_{\mu \nu}(\varphi)=\operatorname{Re}\left(e^{i r \varphi}\left[\zeta\left(a^{\mu}\right)-\zeta\left(a^{\nu}\right)\right]\right)=0 \quad\left(a^{\mu} \neq a^{\nu}\right) .
$$

We set

$$
S_{k}(\delta)=\left\{t \in \boldsymbol{C} \mid \gamma_{k-1}+\delta \leq \arg t \leq \gamma_{k}-\delta\right\} .
$$

Notice that, for $r=1, \gamma_{k}=\varphi_{k} \quad(1 \leq k \leq 2 h)$ where $\varphi_{k}$ is defined as in Subsection 3.2.

b) The functions $x_{\mu}(1 \leq \mu \leq n+m)$ defined by (2.5) have to be substituted here by the functions $x_{\mu}$ defined in (5.5). Here and in the sequel we assume that the sector $N_{k}(\delta)$ is fixed.

According to Subsection. 3.1/b) and c) and (5.5) we have here for $a^{j} \in A$

$$
\begin{aligned}
p\left(t, a^{j}\right) & =\left|\tilde{x}_{\nu_{1}^{j}}(t), \ldots, \tilde{x}_{\nu_{n}^{j}} \cdot(t)\right|=t^{\beta_{\nu 1}^{j}+\ldots+\beta_{\nu_{n}^{j}}}\left(\left|\tilde{v}_{\nu_{1}^{j}}, \ldots ; \tilde{v}_{\nu_{n}^{j}}\right|+\frac{O(1)}{t}\right) \\
p_{\ell \alpha \alpha}\left(t, a^{j}\right) & =t^{\beta_{\nu_{1}^{j}}+\ldots+\beta_{\nu_{n}^{j}}}\left(\left|\tilde{v}_{\nu_{1}^{j}}(\ell, \alpha), \ldots, \tilde{v}_{\nu_{n}^{j}}(\ell, \alpha)\right|+\frac{O(1)}{t}\right)
\end{aligned}
$$

where Landau's O-Symbol $O(1)$ denotes a function of $t$ which is uniformly bounded for $t \in N_{k}(\delta), \delta>0$.

(iv) For $C \in V_{n}\left(C^{n+m}\right)$ we set, generalizing the notations of Section 2,

$$
Y(t ; C)=\left(\hat{y}_{1}(t), \ldots, \hat{y}_{n+m}(t)\right) C=\left(\begin{array}{c}
Q(t) \\
P(t)
\end{array}\right)
$$

with $Q(t) \in \boldsymbol{C}^{n \times n}$ and $P(t) \in \boldsymbol{C}^{m \times n}$. Further we set for $t \in N_{k}(\delta)$ with $\operatorname{det} Q(t) \neq 0$

$$
W_{C}(t)=P(t) Q(t)^{-1}=\left(w_{\ell \alpha}^{C}(t)\right)_{\substack{1 \leq \ell \leq m \\ 1 \leq \alpha \leq n}}
$$

(v) We recall that Assumption 5.1 implies that the number of solutions of equation $(\mathrm{ARE})_{\infty}$ is lesser or equal to $\left(\begin{array}{c}n+m \\ n\end{array}\right)$.

Using the preceding assumptions and notations we infer from the proof of Theorem 1 (see also Remark 1/(ii) and (3.2)) the following corollary. 
Corollary 1 (Fundamental representation formula for the solutions; of equation (5.3)). The function

$$
W_{C}: N_{k}(\delta) \backslash\left\{t \in N_{k}(\delta) \mid \operatorname{det} Q(t)=0\right\} \longrightarrow \boldsymbol{C}
$$

is a solution of the matrix Riccati equation (5.3) with

$$
\begin{aligned}
& w_{\ell \alpha}^{C}(t)= \\
& \qquad \sum_{1 \leq k_{1}<\ldots<k_{n} \leq n+m} e^{\alpha_{k_{1}}(t)+\ldots+\alpha_{k_{n}}(t)} t^{\beta_{k_{1}}+\ldots+\beta_{k_{n}}}\left(\left|v_{k_{1}}(\ell, \alpha), \ldots, v_{k_{n}}(\ell, \alpha)\right|+\frac{O(1)}{t}\right)\left|\begin{array}{c}
c_{k_{1}} \\
\vdots \\
c_{k_{n}}
\end{array}\right|
\end{aligned}
$$

where $\alpha_{j}(t)$ is defined by (5.5).

The following corollary generalizes Lemma 2 . For simplicity we formulate the statements of the corollary for the sector $S_{0}(0) \cup S_{1}(0)$ containing the positive real axis. For the remaining sectors corresponding results hold true.

Corollary 2. Let $\delta>0$ and

$$
C=\left(\begin{array}{c}
c_{1} \\
\vdots \\
c_{n+m}
\end{array}\right) \in V_{n}\left(\boldsymbol{C}^{n+m}\right)
$$

with the following properties:

(i) $D\left(a^{k}, C\right) \neq 0$ and $D\left(a^{j}, C\right)=0$ for $\operatorname{Re} \zeta\left(a^{j}\right)>\operatorname{Re} \zeta\left(a^{k}\right)$.

(ii) $D\left(a^{\mu}, C\right) \neq 0$ and $D\left(a^{j}, C\right)=0$ if $\operatorname{Re}\left(e^{i \varphi} \zeta\left(a^{j}\right)\right)>\operatorname{Re}\left(e^{i \varphi} \zeta\left(a^{\mu}\right)\right)$ for $\gamma_{-1}<\varphi<$ $\gamma_{0}$.

Then the following statements are true.

a) If $\left|\tilde{v}_{\nu_{1}^{k}}, \ldots, \tilde{v}_{\nu_{n}^{k}}\right| \neq 0$, then the solution $W_{C}$ defined by (5.6) and (5.7) has at most a finite number of poles in $S_{1}(\delta)$. Further,

$$
\lim _{S_{1}(\delta) \ni t \rightarrow \infty} W_{C}(t)=W_{0}=\left(w_{\ell \alpha}^{0}\right)_{\substack{\text { i } \\ 1 \leq \alpha \leq n}}
$$

exists and is a solution of equation (ARE) $)_{\infty}$ with

$$
w_{\ell \alpha}^{0}=\frac{\left|v_{\nu_{1}^{j}}(\ell, \alpha), \ldots, v_{\nu_{n}}^{j}(\ell, \alpha)\right|}{\left|\tilde{v}_{\nu_{1}^{j}}, \ldots, \tilde{v}_{\nu_{n}^{j}}\right|}
$$

where the convergence takes place at least at a polynomial rate:

$$
\left|w_{\ell \alpha}^{C}(t)-w_{\ell \alpha}^{0}\right| \leq \frac{\text { const }}{|t|} \quad \text { for } t \in S_{1}(\delta) \text { and }|t| \geq t_{0} .
$$


If in addition $\mu=k$, then $W_{C}$ has at most a finite number of poles in $\tilde{S}(\delta)=\{t \mid \gamma-1+\delta \leq$ $\left.\arg t \leq \gamma_{1}-\delta\right\}$ and the limit (5.8) exists for $\tilde{S}(\delta) \ni t \rightarrow \infty$.

b) If $\mu \neq k$ and $\left|\tilde{v}_{\nu_{1}^{\mu}}, \ldots, \tilde{v}_{\nu_{n}^{\mu}}\right| \neq 0 \neq\left|\tilde{v}_{\nu_{1}^{k}}, \ldots, \tilde{v}_{\nu_{n}^{k}}\right|$, then $W_{C}$ has an infinite number of poles in $B^{\delta}=\left\{t \in \boldsymbol{C} \mid \gamma_{0}-\delta \leq \arg t \leq \gamma_{0}+\delta\right\}$ and at most a finite number of poles in $S_{0}(\delta) \cup S_{1}(\delta)$. Further,

$$
\lim _{S_{0}(\delta) \ni t \rightarrow \infty} W_{C}(t)=W_{1}=\left(w_{\ell \alpha}^{1}\right)_{\substack{1 \leq \ell \leq m \\ 1 \leq \alpha \leq n}}
$$

with

$$
w_{\ell \alpha}^{1}=\frac{\left|v_{\nu_{1}^{\mu}}(\ell, \alpha), \ldots, v_{\nu_{n}^{\mu}}(\ell, \alpha)\right|}{\left|\tilde{v}_{\nu_{1}^{\mu}}, \ldots, \tilde{v}_{\nu_{n}^{\mu}}\right|} .
$$

c) If $\left|\tilde{v}_{\nu_{1}^{k}}, \ldots, \tilde{v}_{\nu_{n}^{k}}\right|=0$ and $\left|v_{\nu_{1}^{k}}(\ell, \alpha), \ldots, v_{\nu_{n}^{k}}(\ell, \alpha)\right| \neq 0$, then

$$
\lim _{S_{1}(\delta) \ni t \rightarrow \infty} w_{\ell \alpha}^{C}(t)=\infty
$$

For the investigation of the behaviour of the remaining elements $w_{i \nu}^{C}(t)$ one needs representations of the solutions $\dot{\hat{y}}_{\mu}$ which are more precise than those given by (5.5).

Proof. a) Since the eigenvalues $\lambda_{1}, \ldots, \lambda_{n+m}$ are simple it follows as with the proof of Lemma 3 that

$$
D\left(a^{\nu}, C\right)=0 \quad \text { if } a^{\nu} \notin\left\{a^{\mu}, a^{k}\right\} \text { and } \zeta\left(a^{\nu}\right) \in\left\{\zeta\left(a^{\mu}\right), \zeta\left(a^{k}\right)\right\} .
$$

Let $t \in S_{1}(\delta)$ and $\left|\tilde{v}_{\nu_{1}^{k}}, \ldots, \tilde{v}_{\nu_{n}^{k}}\right| \neq 0$. Dividing nominator and denominator of the representation formula for $w_{\ell \alpha}^{C}(t)$ (see Corollary 1 ) by

$$
e^{\alpha_{\nu_{1}^{k}}(t)+\ldots+\alpha_{\nu_{n}^{k}}(t)} t^{\beta_{k_{1}}+\ldots+\beta_{k_{n}}}\left|\begin{array}{c}
c_{k_{1}} \\
\vdots \\
c_{k_{n}}
\end{array}\right|
$$

we infer, using (5.13), that there exist $t_{0}>0$ and $\varepsilon_{0}(\delta)>0$ with

$$
w_{\ell \alpha}^{C}(t)=\frac{\left|v_{\nu_{1}^{k}}(\ell, \alpha), \ldots, v_{\nu_{n}^{k}}(\ell, \alpha)\right|+\frac{O(1)}{t}+O\left(e^{-\varepsilon_{0}(\delta)}\right)}{\left|\tilde{v}_{\nu_{1}^{k}}, \ldots, \tilde{v}_{\nu_{n}^{k}}\right|+\frac{O(1)}{t}+O\left(e^{-\varepsilon_{0}(\delta)}\right)}
$$

for $t \geq t_{0}$. This implies (5.8) - (5.10).

If $\mu=k$, then (5.14) is valid for $\gamma-1+\delta \leq \arg t \leq \gamma_{1}-\delta$, which proves the last statement of item a). If $\mu \neq k$, then (under the assumptions of item b)) for $t \in S_{0}(\delta)$ the terms containing the factor $e^{\alpha_{\nu}^{\mu}(t)+\ldots+\alpha_{\nu}^{\mu}(t)}$ are the dominant terms in the representation formula for $W_{C}(t)$. Hence we infer from the properties of asymptotic exponential polynomials (see [4: ???] and [16: ???]) that $W_{C}$ has an infinite number of poles in $B^{\delta}$.

The remaining assertions of Corollary 2 are obtained similarly from Corollary 1 
Remark 4. (i) Obviously any solution of equation (ARE) $)_{\infty}$ can be represented as a limit of the form (5.8), and from Subsection 3.5 it follows that equation (ARE) $)_{\infty}$ has at $\operatorname{most}\left(\begin{array}{c}n+m \\ n\end{array}\right)$ solutions.

(ii) If $A_{0}$ has multiple eigenvalues, then the behaviour of the solutions of equation (5.3) may be different from that described in Corollary 2. In particular there may be solutions of equation (5.3) having an infinite number of poles in the sector $S_{k}(\delta)$. These poles have at most logarithmic density, which means that for $\delta>0$ the number of the poles of any solution of equation (5.3) in $S_{k}(\delta) \cap\{t \in C|| t \mid \leq R\}$ is at most of the order $O(\log R)$ for $R \rightarrow \infty$ (see [12] for further details).

(iii) If $\gamma_{0}<0<\gamma_{1}$, then Corollary 2 gives a description of the solutions of equation (5.3) for $t>0$. If $\gamma_{0}=0$, then it is possible to generalize some of the results obtained in Section 4 for the autonomous Riccati equation $(\mathrm{RDE})_{\mathbb{R}}$ to the non-autonomous case. We give the following example which corresponds to Remark 3/(ii) and which is an easy consequence of Corollary 1.

Corollary 3. Let $\operatorname{Re} \lambda_{m}<\operatorname{Re} \lambda_{m+1}$,

$$
\left(\begin{array}{l}
A \\
B
\end{array}\right)=\left(v_{m+1}, \ldots, v_{m+n}\right) \quad \text { with } A=\left(\tilde{v}_{m+1}, \ldots, \tilde{v}_{m+n}\right) \text { and }|A| \neq 0
$$

Then $W^{*}=B A^{-1}$ is the dichotomic solution of equation (5.2) and any solution $W_{C}$ (defined by (5.6) and (5.7) for $k=1$ ) with $\left|c_{m+1} \cdots c_{m+n}\right|^{\top} \neq 0$ converges for $0<t \rightarrow \infty$ at least at a polynomial rate to $W^{*}$.

\section{References}

[1] Abou-Kandil, A., Freiling, G. and G. Jank: Necessary conditions for constant solutions of coupled Riccati equations in Nash games. Syst. Control Letters 21 (1993), 295 - 306.

[2] Anderson, B. D. O. and D. J. Clements: Polynomial factorization via the Riccati equation. SIAM J. Appl. Math. 31 (1976), 179 - 205.

[3] Balser, W., Jurkat, W. B. and D. A. Lutz: Birkhoff invariants and Stokes' multipliers for meromorphic linear differential equations. J. Math. Anal. Appl. 71 (1979), 48 - 94.

[4] Boese, F. G.: Zur Lage der Nullstellen von Exponentialpolynomen und zur Stabilität von Exponentialpolynomen. Habilitationsschrift. Universität Hamburg 1989.

[5] Brockett, R. W.: Finite dimensional linear systems. New York: John Wiley 1970.

[6] Bucy, R. S.: Structural stability for the Riccati equation. SIAM J. Control Opt. 13 (1975), $749 \cdot 753$.

[7] Callier, F. M. and J. L. Willems: Criterion for the convergence of the solution of the Riccati differential equation. IEEE Trans. Aut. Control AC-26 (1981), 1232 - 1242.

[8] Freiling, G. and G. Jank: Matrix Ricatti Equations. Universität Duisburg: Schriftenreihe des FB Mathematik SM-DU 193 (1993), 26 pp.

[9] Griffiths, P. and J. Harris: Principles of Algebraic Geometry. New York: John Wiley 1978. 
[10] Hermann, R. and C. Martin: Lie and Morse theory for periodic orbits of vector fields and matrix Riccati equations. Part I: General Lie-theoretic methods. Math. Systems Theory 15 (1982), 277 - 284.

[11] Hermann, R. and C. Martin: Lie and Morse Theory for periodic orbits of vector fields and matrix Riccati equations. Part II. Math. Systems Theory 16 (1983), 297 - 306.

[12] Jank, G.: Asymptotic distribution of singularities of solutions of matrix-Riccati-differential equations. J. Austral. Math. Soc. (Ser. B) 34 (1992), 112 - 131.

[13] Jurkat, W. B.: Meromorphe Differentialgleichungen. Berlin: Springer - Verlag 1978.

[14] Knobloch, H. W. and H. Kwakernaak: Lineare Kontrolltheorie. Berlin: Springer - Verlag 1988.

[15] Kucera, V.: Algebraic Riccati equation: Symmetric and definite solutions. In: Lect. Notes of the Workshop on "The Riccati Equation in Control, Systems and Signal" (ed.: S. Bittanti). Bologna: Pitagora Editrice 1989, pp. 73 - 77.

[16] Langer, R. E.: On the zeros of exponential sums and integrals. Bull. Amer. Math. Soc. 37 (1931), $213-239$.

[17] Màrtensson, K.: On the matrix Riccati equation. Inform. Sci. 3 (1971), 17 - 49.

[18] Medanic, J.: Geometric properties and invariant manifolds of the Riccati equation. IEEE Trans. Automat. Control AC-27 (1982), $670-677$.

[19] Meyer, H. B.: The matrix equation $A Z+B-Z C Z-Z D=0$. SIAM J. Appl. Math. 30 (1976), 136 - 142.

[20] Potter, J. E.: Matrix quadratic solutions. SIAM J. Appl. Math. 14 (1966), 496 - 501.

[21] Reid, W. T.: Riccati Differential Equations. New York: Acad. Press 1972.

[22] Schneider, C. R.: Global aspects of the matrix Riccati equation. Math. Systems Theory 7 (1973), $281-286$.

[23] Shayman, M. A.: On the variety of invariant subspaces of a finite-dimensional linear operator. Trans. Amer. Math. Soc. 274 (1982), 721 - 747.

[24] Shayman, M. A.: Geometry of the algebraic Riccati equation. Part I. SIAM J. Control Opt. 21 (1983), 375 - 394.

[25] Shayman, M. A.: Geometry of the algebraic Riccati equation. Part II. SIAM J. Control Opt. 21 (1983), 395 - 409.

[26] Shayman, M. A.: On the periodic solutions of the matrix Riccati equation. Math. Systems Theory 16 (1983), $267-287$.

[27] Shayman, M. A.: Phase portrait of the matrix Riccati equation. SIAM J. Control and Opt. 24 (1986), 1 - 65.

[28] Telford, M. A. and J. B. Moore.: On the existence of solutions to nonsymmetric algebraic Riccati equations. Lect. Notes of the Workshop on "The Riccati Equation in Control, Systems and Signal" (ed.: S. Bittanti). Bologna: Pitagora Editrice 1989, pp. 83 - 86. 